\title{
Disk-Outflow Models as Applied to High Mass Star Forming Regions through Methanol and Water Maser Observations
}

\author{
Hontas F. Farmer \\ Department of Physical Science Richard J. Daley College, City Colleges of Chicago, Chicago, IL, USA \\ Email: hfarmer@ccc.edu
}

Received 24 August 2014; revised 20 September 2014; accepted 14 October 2014

Copyright (C) 2014 by author and Scientific Research Publishing Inc.

This work is licensed under the Creative Commons Attribution International License (CC BY).

http://creativecommons.org/licenses/by/4.0/

c) (i) Open Access

\section{Abstract}

As the recent publication by Breen et al. (2013) found, Class II methanol masers are exclusively associated with high mass star forming regions. Based on the positions of the Class I and II methanol and $\mathrm{H}_{2} \mathrm{O}$ masers, UC H II regions and $4.5 \mu \mathrm{m}$ infrared sources, and the center velocities ( $\left.v_{\mathrm{LSR}}\right)$ of the Class I methanol and $\mathrm{H}_{2} \mathrm{O}$ masers, compared to the $v_{\mathrm{LSR}}$ of the Class II methanol masers, we propose three disk-outflow models that may be traced by methanol masers. In all three models, we have located the Class II methanol maser near the protostar, and the Class I methanol maser in the outflow, as is known from observations during the last twenty years. In our first model, the $\mathrm{H}_{2} \mathrm{O}$ masers trace the linear extent of the outflow. In our second model, the $\mathrm{H}_{2} \mathrm{O}$ masers are located in a circumstellar disk. In our third model, the $\mathrm{H}_{2} \mathrm{O}$ masers are located in one or more outflows near the terminating shock where the outflow impacts the ambient interstellar medium. Together, these models reiterate the utility of coordinated high angular resolution observations of high mass star forming regions in maser lines and associated star formation tracers.

\section{Keywords}

Masers, Methanol, Massive Star Formation, Stars: Evolution, Stars: Distances

\section{Introduction}

Understanding the process of the formation of high mass stars with masses about eight times that of our Sun, or greater, remains a challenge in modern astronomy. High mass stars are important because they inject matter and energy into the interstellar medium of galaxies throughout their lifetimes. Observing high mass star formation is difficult, though, because massive star forming regions are farther away than their low mass counterparts, high 
mass stars form in clusters, and begin the process of nuclear fusion even as they are accreting matter. One of the key issues is whether high mass star formation is a scaled-up version of the low mass star formation process, complete with disk and outflows, or whether high mass stars form by coalescence of low mass stars. Being compact and bright sources, masers offer a unique opportunity to peer into the deep interior of high mass star forming regions at high angular resolution. In this paper, we use data on methanol and water masers from the literature, along with other associated star formation tracers, to gain insight into the high mass star formation process by investigating whether disk-outflow systems are compatible with these maser data. The exclusive association of Class II methanol masers with high mass protostars was shown by [1]. In the thesis [2], a similar analysis was carried out for a larger selection of regions. This paper is intended as a journal publishable write up of [2] updated with Breen's results. In this section, we begin with a discussion of low mass star formation in $\S 1.1$, high mass star formation in §1.2, and masers and maser observations in $§ 1.3$.

\subsection{Low Mass Star Formation}

Over the last fifty years, astronomers have gained a fairly good understanding of the basic process of low mass star formation. Star formation is initiated when dense cores inside molecular clouds become gravitationally unstable and collapse. Depending on the size of the core, one or more stars will be formed. Small cores may form a single star, larger cores will fragment and form multiple stars (some authors use the term "clumps" for larger cores).

The exact details of the process of collapse are model dependent, but the following are generally common to most models [3], Gravitational collapse builds up the central density in the core of the cloud or cloud fragment. The energy generated by the gravitational collapse is radiated away because the cloud remains optically thin (i.e., transparent to radiation); as a result, during the initial stages of the collapse, most models treat the cloud as isothermal. The isothermal collapse phase produces a central condensation of matter and ends with the formation of a stable object at the center called a protostar, surrounded by a gaseous envelope. With the increasing density in the gaseous envelope, it becomes harder for the energy released by gravitational contraction to escape from the star, as a result of which the internal temperature of the protostar rises. The protostar then enters its main accretion phase, in which it builds up its mass from a surrounding infalling envelope and accretion disk, even as it continues to get hotter. Some of the accreted material is ejected along opposite directions perpendicular to the accretion disk, and this is known as a bipolar outflow. These outflows are believed to help in carrying away the excess angular momentum of the infalling matter; this is necessary because of the so-called "angular momentum problem" in star formation. This problem arises because while rotation is not a significant source of support in molecular clouds against gravity, a decrease of just a factor of 100 in size (while conserving angular momentum) increases the rotational energy to the point where it can support the cloud against gravitational collapse. Yet, since decreases by factors of $10^{5}$ or greater in size are required for a core to become a star, if it were the case that a decrease of just $10^{2}$ were enough to bring the rotational energy into equilibrium with gravitational energy, then stars could never form. Mechanisms have been sought for how to get rid of the angular momentum during collapse, and bipolar outflows and the magnetic fields that are present in these outflows are believed to carry away the excess angular momentum, but the exact mechanism is still not fully understood.

When the protostar has accumulated most of its final mass (that it will have during its lifetime as a main sequence star) it is generally known as a premain-sequence (PMS) star, although the use of the term varies among authors (McKee 2007). Such stars continue to supply most of their luminosity (i.e., the energy output in, e.g., ergs $\cdot \mathrm{s}^{-1}$ ) by contracting and releasing gravitational potential energy, but now their luminosities and surface temperatures increase. Eventually, the interior becomes hot enough to initiate hydrogen fusion, and this takes over as the cause of the luminosity of the star. The star soon comes into hydrostatic equilibrium between the outward thermal pressure and the inward gravitational pull and reaches its main sequence phase, in which it will spend $90 \%$ of its life span, steadily fusing hydrogen to helium.

\subsection{High Mass Star Formation}

The process of high mass star formation is not as well understood as low mass star formation. For a number of reasons high mass stars constitute a very difficult observational and theoretical problem. High mass star forming regions are located farther from us than low mass star forming regions; the nearest low mass star forming region is Ophiuchus at a distance of $120 \mathrm{pc}$ [4], whereas the nearest high mass star forming region in Orion is 500 pc 
away ([5]); high mass stars usually form in clusters, and because of their high mass, they begin fusing hydrogen even as they are accreting material. It is difficult to find an isolated high mass star in the process of forming with which to study the process. If we observe an outflow, it is often difficult to figure out which high mass protostar the outflow is coming from, because the protostars are so close together. Moreover, high mass star forming regions are obscured by more gas and dust than low mass star forming regions ([6]).

Theoretical studies of this process must address how accretion can continue in the face of the tremendous outward radiation pressure generated by the onset of hydrogen fusion.

\subsection{Maser Observations of High Mass Star Forming Regions}

Observations of maser lines are ideal for studying high mass star forming regions at high angular resolution, as masers are compact and bright sources. There are two varieties of methanol maser, with Class II masers exclusively associated with high mass star forming regions as shown by Breen2013 in their analysis of two regions (G328.385 + 0.131, G10.10 + 0.73).

The division of methanol masers into two classes was originally based on association: Class II masers are associated with protostars, and Class I masers are found in outflows ([7]). Today, we know there are differences in the pumping mechanism as well; Class II methanol masers are believed to be radiatively pumped, while Class I masers are pumped collisionally. This would also explain why Class II masers are found near high mass protostars where they are pumped by the intense radiation from the protostar ([8]), and why Class I masers are found in outflows where they are pumped by collision at the interfaces of shock fronts between the outflowing material and the ambient interstellar material ([9]). The most well known Class II methanol masers occur at frequencies of $6.7 \mathrm{GHz}$ and $12.2 \mathrm{GHz}$, whereas Class I methanol masers occur at $44 \mathrm{GHz}, 95 \mathrm{GHz}$, and $36 \mathrm{GHz}$.

Figure 1 is a diagram which shows the energy levels of type $\mathrm{E}$ methanol for different values of $\mathrm{K}$, where $\mathrm{K}$ is the projection of the total angular momentum $\mathrm{J}$ onto the principal axes of the molecule. There are two species of methanol, E and A, differentiated by the spin orientations of their hydrogen atoms. This figure was derived from one due to [10] and used with the original authors permission. Knowing the spectra of molecules on Earth, and the laws of Doppler shift allow us to figure out what elements star forming clouds are made of. Knowing how these molecules are excited lets us figure out the interactions of these gaseous regions.

\section{Data Sources and Collection}

[2] began by looking for high angular resolution data on Class I and Class II methanol masers in star forming regions; angular resolution is defined and discussed in the next paragraph. [11] is a compiled catalog of all the known Class I methanol masers. From the 206 masers in their list, we picked the $44 \mathrm{GHz}$ methanol masers that had been observed at high angular resolution with the the Karl G. Jansky Very Large Array (VLA); there were 32 sources of this kind. For each of these 32 maser sources, we searched the literature for Class II methanol masers within a 1' radius of the position listed in the [11] catalog. Since $\mathrm{H}_{2} \mathrm{O}$ masers are also signposts of star formation, we searched the literature for $\mathrm{H}_{2} \mathrm{O}$ masers near these Class I and II methanol masers. Recently, [12] have shown that $4.5 \mu \mathrm{m}$ infrared sources are associated with high mass star formation, so we also looked for such sources near the Class I and II methanol masers. Finally, young high mass stars are known to form ultra compact (UC) ionized hydrogen (H II) regions by ionizing the hydrogen around them, so we also looked for data on UC H II regions.

We wanted to work with the highest angular resolution data available for our sources. Therefore, we preferred to use data taken with interferometric telescopes like the VLA wherever available. The angular (or spatial) resolution of a telescope is given by the ratio of the wavelength of observation to the diameter of the telescope. When signals from two radio telescope antennas are combined, the effective diameter of the telescope is the distance between the antennas. Therefore, by locating telescopes at large distances from each other as in the VLA, interferometers achieve much greater resolution than a single dish radio telescope. For example, the angular resolution of a single dish telescope of diameter $25 \mathrm{~m}$ would be about 60" if it were observing at a frequency of $44 \mathrm{GHz}$. By way of comparison, [13] were able to obtain a much higher angular resolution of 5", i.e., over ten times better, in their VLA observations of $44 \mathrm{GHz}$ methanol masers discussed below.

We will now discuss the characteristics of the observed data, specifically, the papers we consulted, the telescope and observing parameters used to observe these data, and any other relevant information. 


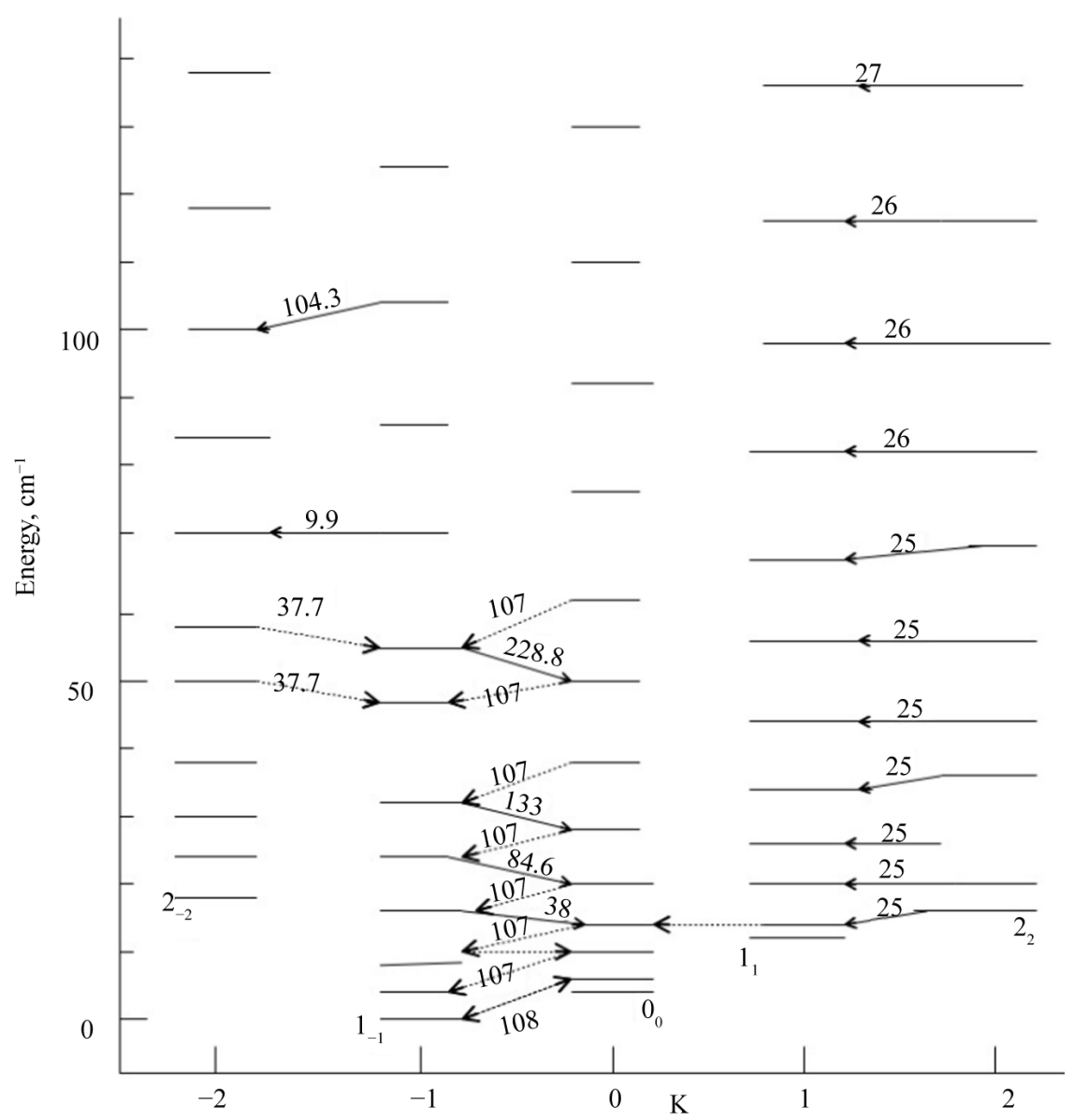

Figure 1. Diagram showing the energy levels of type E methanol for different values of K, where $\mathrm{K}$ is the projection of the total angular momentum $\mathrm{J}$ onto the principal axes of the molecule. There are two species of methanol, E and A, differentiated by the spin orientations of their hydrogen atoms. This figure was derived from one due to [10] and used with the original authors permission.

\subsection{The Class I Methanol Maser Data}

The positions of the $44 \mathrm{GHz}$ Class I methanol masers were taken from the [11] catalog. Most of the positions listed in this catalog were taken from VLA observations reported in [13], from which we obtained information on the center velocities $\left(v_{\mathrm{LSR}}\right)$ and intensities of the methanol masers. [13] was a survey of fortyfour star forming regions in search of $44 \mathrm{GHz}$ Class I methanol masers with the goal of investigating the relationship between such masers and shocked molecular gas. The angular resolution of this survey was about $0.5 "$, and the velocity resolution was $0.17 \mathrm{~km} \cdot \mathrm{s}^{-1}$ (corresponding to a frequency resolution of about $24 \mathrm{kHz}$ ). The idea of frequency resolution comes from the finite bandwidth of telescope receivers that must be sampled in discrete intervals. So, for example, [13]'s survey used a frequency set up in which the bandwidth was $3.125 \mathrm{MHz}$ sampled with 128 channels, to give a frequency resolution of $24 \mathrm{kHz}$. However, astronomers like to work with velocities because they can be connected more easily to the physical motions of the source(s) in the sky. For Galactic observations such as those studied in this paper, one would convert from one to the other using the non relativistic Doppler effect: $\Delta v / c=\Delta f / f$, where $\Delta f$ is the shift in frequency from the observed frequency, $f$. The root mean square (rms) noise in the maser spectra line profiles, usually measured as one fifth of the peak-to-peak value in the line observed in a source-free region of the sky, was typically $40 \mathrm{mJy}^{\text {beam }}{ }^{-1}$, so they were able to detect masers down to a median 5- $\sigma$ limit of $0.2 \mathrm{Jy}$. For several sources, [13] found evidence for a spatial correlation between the $44 \mathrm{GHz}$ masers and shocked molecular gas in agreement with the view that these masers are produced by collisional pumping in molecular outflows. 


\subsection{The Class II Methanol Maser Data}

The Class II methanol maser data were taken from a catalog of $6.7 \mathrm{GHz}$ Class II methanol masers compiled by [14]. This catalog contains 519 sources compiled from 62 references in the literature. [14] found that Class II methanol masers trace the molecular ring of our galaxy where massive OB star associations are found, in agreement with the idea that methanol masers are clearly associated with high mass star formation.

[14] relied heavily on [15], which was a survey of thirty-six sites of $6.7 \mathrm{GHz}$ methanol masers and forty sites of $1.7 \mathrm{GHz} \mathrm{OH}$ masers, conducted with the Australia Telescope Compact Array (ATCA). The purpose of this survey was to study the relationship between $\mathrm{OH}$ and $\mathrm{CH}_{3} \mathrm{OH}$ masers and the evolution of massive stars. The angular resolution of the methanol maser observations was about 1.5 ", the frequency resolution was $0.97 \mathrm{kHz}$, and the rms noise was $60 \mathrm{mJy} \cdot$ beam $^{-1}$.

For more recent data in the literature, we used [16] and [17]. [16] observed 57 Class II methanol masers at 6.7 $\mathrm{GHz}$ with the Multi-Element Radio-Linked Interferometer Network (MERLIN, Jodrell Bank) with an angular resolution of 60 milliarcseconds (mas), a frequency resolution of $3 \mathrm{kHz}\left(\equiv 0.13 \mathrm{~km} \cdot \mathrm{s}^{-1}\right)$, and an rms noise of about $35 \mathrm{mJy} \cdot$ beam $^{-1}$. They found very close correspondence between methanol masers and $24 \mu \mathrm{m}$ mid-infrared sources, lending further support to theoretical models that predict methanol masers are pumped by infrared dust emission in the vicinity of high-mass protostars. [17] observed $44 \mathrm{GHz}$ Class I and $6.7 \mathrm{GHz}$ Class II methanol masers in 20 sources with the VLA. These twenty sources were selected based on $4.5 \mu \mathrm{m}$ Spitzer infrared images (see §2.5). The angular resolution of the $44 \mathrm{GHz}$ VLA observations was $0.5 "$ - 1", the frequency reslution was $24 \mathrm{kHz}\left(\equiv 0.17 \mathrm{~km} \cdot \mathrm{s}^{-1}\right)$, and the rms noise was about $25 \mathrm{mJy} \cdot$ beam $^{-1}$. The angular resolution of the $6.7 \mathrm{GHz} \mathrm{VLA}$ observations was 2" - 4", the frequency resolution was $3.05 \mathrm{kHz}\left(\equiv 0.14 \mathrm{~km} \cdot \mathrm{s}^{-1}\right)$, and the rms noise was about 27 mJy beam $^{-1}$. [17] found a strong association between the $4.5 \mu \mathrm{m}$ selected objects called EGOs (for "Extended Green Objects”) and Class II masers, and a widespread distribution of $44 \mathrm{GHz}$ Class I masers in outflows, indicating that EGOs are young, high mass star forming objects that drive active outflows.

\subsection{The $\mathrm{H}_{2} \mathrm{O}$ Maser Data}

Most of the $\mathrm{H}_{2} \mathrm{O}$ maser data discussed in this paper were taken from [18] who obtained images and spectra for $21 \mathrm{H}_{2} \mathrm{O}$ maser sources in the vicinity of UC H II regions by observing with the VLA at 22 GHz. The angular resolution of their survey was 0.4 ", the frequency resolution was $24 \mathrm{kHz}\left(\equiv 3.5 \mathrm{~km} \cdot \mathrm{s}^{-1}\right)$, and the typical RMS noise was $30 \mathrm{mJy} \cdot$ beam $^{-1}$.

\subsection{The UC H II Region Data}

Almost all of the UC H II region data in this paper were taken from [19], who surveyed seventy-five UC H II regions with the VLA at frequencies of $4.9 \mathrm{GHz}$ and $14.9 \mathrm{GHz}$. The goal of this survey was to understand the morphology and characteristics of the selected H II regions. The $14.9 \mathrm{GHz}$ observations used for this paper have an angular resolution of approximately $0.4 "$ and an rms noise of about $0.32 \mathrm{mJy} \cdot \mathrm{beam}^{-1}$. In addition, the data for one UC H II region in G45.47 + 0.07 were obtained from [20], who observed 659 high mass star forming candidate regions with the VLA at $4.9 \mathrm{GHz}$ in order to detect UC H II regions. The angular resolution of their observations was about 1.5", and the rms noise was about $0.2 \mathrm{mJy} \cdot$ beam $^{-1}$. They identified 391 UC H II regions in this sample.

\subsection{The $4.5 \mu \mathrm{m}$ Infrared Data}

The $4.5 \mu \mathrm{m}$ infrared data used in this paper were taken from the GLIMPSE catalog of the online Spitzer archive (where GLIMPSE stands for Galactic Legacy Infrared Midplane Survey Extraordinaire). The GLIMPSE catalog ([21]) was compiled from observations taken with the Infrared Array Camera (IRAC) on board the Spitzer Space Telescope, which recorded data at wavelenghts of $3.6 \mu \mathrm{m}, 4.5 \mu \mathrm{m}, 5.8 \mu \mathrm{m}$, and $8.0 \mu \mathrm{m}$. The angular resolution of GLIMPSE images is about 1.2". We use the $4.5 \mu \mathrm{m}$ data because [22] have found that extended emission at $4.5 \mu \mathrm{m}$ is associated with high mass star forming regions. This wavelength is usually colored green in most false color infrared images, hence the term extended “green” objects (EGO’s).

\section{Results}

We present the results. In §3.1, we discuss global features of our data. In §3.2, we present a discussion of eight 
individual regions for which high resolution data were available for Class I and Class II methanol masers and associated tracers. In §3.11, we present results for the regions for which high angular resolution data were available only for Class I and Class II methanol masers.

\subsection{Masers and Associated Objects}

The data for this project were obtained by searching the literature for high angular resolution data on thirty star forming regions known to contain Class I and Class II methanol masers. In addition to these types of masers, we searched the literature for high angular resolution data on associated $\mathrm{H}_{2} \mathrm{O}$ masers, ultra compact (UC) ionized hydrogen (H II) regions, and $4.5 \mu \mathrm{m}$ Spitzer infrared images in these thirty star forming regions. As shown in Figure 2, all of these data were available for eight, or $27 \%$ of the thirty regions. For $13 \%$ of the regions, $\mathrm{H}_{2} \mathrm{O}$ maser data, which were crucial to the analyses we intended to perform, were not found, while data on methanol masers, and UC H II regions were found. For the remaining $60 \%$ of the regions, high angular resolution data were present for the methanol masers only.

\subsection{Discussion of Individual Regions}

For those eight regions for which data are available in all the tracers listed in §3.1, plots have been produced showing the positions of the Class I and II methanol masers, $\mathrm{H}_{2} \mathrm{O}$ masers, UC H II regions and near infrared sources. Diagrams showing the center velocities $\left(v_{\mathrm{LSR}}\right)$ and intensities of the methanol and $\mathrm{H}_{2} \mathrm{O}$ maser spectral lines were also produced for these regions.

With these plots of morphology and spectra we seek to address the following issues:

1) What is a typical range of projected distances between Class I and II methanol masers?

2) How do the center velocities $\left(v_{\mathrm{LSR}}\right)$ of the $\mathrm{H}_{2} \mathrm{O}$ masers compare to that of the Class I and II methanol masers?

3) How are $\mathrm{H}_{2} \mathrm{O}$ masers arranged in relation to these methanol masers? In particular, are there any arrangements that suggest a known configuration like an outflow or disk?

The eight regions will now be discussed individually.

\subsection{Region G9.62 + 0.19}

Figure 3 shows the Class II methanol maser and the Class I methanol maser in G9.62 + 0.19, together with other



Figure 2. Pie chart showing the type and quality of available data. In addition to Class I and Class II methanol masers, we looked for data on $\mathrm{H}_{2} \mathrm{O}$ masers, UC H II regions, and $4.5 \mu \mathrm{m}$ infrared sources. All of these data were available for eight, or $27 \%$, of the regions. For $13 \%$ of the regions, $\mathrm{H}_{2} \mathrm{O}$ maser data were not found. For the remaining $60 \%$ of the regions, high resolution data were present for the methanol masers only. 


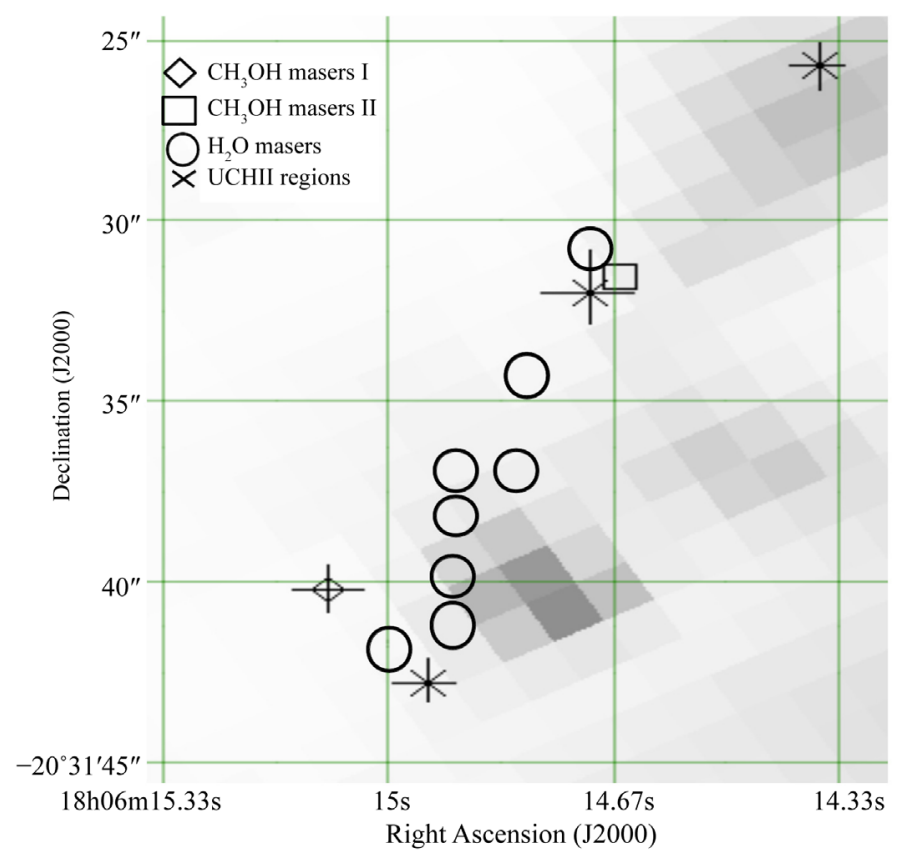

\begin{abstract}
Figure 3. Figure showing the Class II and the Class I methanol maser and other sources of interest in region G9.62 +0.19 . The position of the Class I methanol maser is indicated by a diamond and the Class II methanol maser by a square. The open circles mark the positions of the $\mathrm{H}_{2} \mathrm{O}$ masers and the $\times$ 's mark the peaks of the UC H II regions. In all cases, positional uncertainties are either equal to or smaller than the sizes of the markers themselves or are marked by a cross, if larger than the marker sizes. The grayscale shows the $4.5 \mu \mathrm{m}$ Spitzer infrared image and the intensity range is between $1.61 \mathrm{MJy} \cdot \mathrm{sr}^{-1}$ and $938 \mathrm{MJy} \cdot \mathrm{sr}^{-1}$. Per usual astronomical convention for images, north is upward and east is to the left in this, and all other images in this paper.
\end{abstract}

sources of interest. To the northwest of the Class I maser position is a Class II methanol maser, whose position is marked by a square in Figure 3. Following usual astronomical convention, east is to the left in Figure 3, and all other images in this paper. The Class I and Class II masers are separated by about 10.7"; at a distance of 5.2 kiloparsec (kpc) to G9.62 + 0.19 ([23]), this is equivalent to about 0.27 pc. The $\mathrm{H}_{2} \mathrm{O}$ masers in this source are arranged along a narrow, almost linear, structure (Figure 3). There are three UC H II regions in this figure ([24]). There is a UC H II region about 3.4" to the southwest of the Class I methanol maser, and a second UC H II region that is almost coincident with the Class II methanol maser. The grey scale shows the $4.5 \mu \mathrm{m}$ infrared Spitzer image. About 3.8" west of the Class I maser position lies a peak in this $4.5 \mu \mathrm{m}$ image. The positions of these sources, together with the telescopes used to observe them and the angular resolution (in terms of synthesized beam) of the observations, are listed in Table 1.

The center velocities $\left(v_{\text {LSR }}\right)$ and intensities of the Class I and Class II methanol masers and $\mathrm{H}_{2} \mathrm{O}$ masers have been plotted in Figure 4. In this, and all other such figures, the $v_{\text {LSR }}$ of the Class II methanol maser will be used as a reference; masers with $v_{\mathrm{LSR}}$ larger than the Class II methanol maser will be considered to be redshifted, and masers with $v_{\text {LSR }}$ smaller than the Class II methanol maser will be considered to be blueshifted. Six of the eight $\mathrm{H}_{2} \mathrm{O}$ masers in G9.62 + 0.19 are at larger center velocities $\left(v_{\mathrm{LSR}}\right)$ than the center velocity of the Class II methanol maser, that is, they are redshifted with respect to the Class II methanol maser. The Class I methanol maser is also redshifted (i.e., at larger $v_{\mathrm{LSR}}$ ) with respect to the Class II methanol maser. A ninth $\mathrm{H}_{2} \mathrm{O}$ maser with a center velocity of $25 \mathrm{~km} \cdot \mathrm{s}^{-1}$ is outside the range of the plot in Figure 4.

\title{
3.4. Region G10.47 + 0.03
}

Figure 5 shows the Class II methanol maser and the Class I methanol maser in G10.47 + 0.03, together with other sources of interest. The Class II methanol maser is located to the southeast of the Class I maser position, and its 
Table 1. Positions and associated information for sources associated with G9.62 + 0.19.

\begin{tabular}{|c|c|c|c|c|c|c|}
\hline Source type & R.A. (J2000) & Dec. (J2000) & Observing & Synthesized & Kinematic & \\
\hline & (h m s) & $\left(0^{\prime} \prime \prime\right)$ & Telescope & Beam & $v_{\mathrm{LSR}}\left(\mathrm{km} \cdot \mathrm{s}^{-1}\right)$ & Intensity (Jy) \\
\hline $44 \mathrm{GHz}$ Class $\mathrm{I} \mathrm{CH}_{3} \mathrm{OH}$ maser $^{\mathrm{a}}$ & 180615.1 & -203140 & VLA $^{\mathrm{b}}$ & $1.1^{\prime \prime} \times 1.1^{\prime \prime}$ & 3.7 & 2.1 \\
\hline 12.2 GHz Class II $\mathrm{CH}_{3} \mathrm{OH}$ maser ${ }^{\mathrm{c}}$ & 180614.7 & -203132 & VLBA $^{d}$ & $0.001 " \times 0.001 "$ & 1.3 & 5500 \\
\hline $22 \mathrm{MHz} \mathrm{H}_{2} \mathrm{O}$ maser $^{\mathrm{e}}$ & 180614.7 & -203131 & VLA & $0.64 " \times 0.33^{\prime \prime}$ & 0.10 & 1.3 \\
\hline $22 \mathrm{MHz} \mathrm{H}_{2} \mathrm{O}$ maser & 180614.8 & -203134 & VLA & $0.64 " \times 0.33^{\prime \prime}$ & -9.8 & 2.9 \\
\hline $22 \mathrm{MHz} \mathrm{H}_{2} \mathrm{O}$ maser & 180614.8 & -203137 & VLA & $0.64^{\prime \prime} \times 0.33^{\prime \prime}$ & 5.3 & 17 \\
\hline $22 \mathrm{MHz} \mathrm{H}_{2} \mathrm{O}$ maser & 180614.9 & -203137 & VLA & $0.64^{\prime \prime} \times 0.33^{\prime \prime}$ & 6.0 & 2.2 \\
\hline $22 \mathrm{MHz} \mathrm{H}_{2} \mathrm{O}$ maser & 180614.9 & -203138 & VLA & $0.64 " \times 0.33^{\prime \prime}$ & 7.3 & 1.5 \\
\hline $22 \mathrm{MHz} \mathrm{H}_{2} \mathrm{O}$ maser & 180614.9 & -203140 & VLA & $0.64^{\prime \prime} \times 0.33^{\prime \prime}$ & 7.0 & 6.4 \\
\hline $22 \mathrm{MHz} \mathrm{H}_{2} \mathrm{O}$ maser & 180614.9 & -203141 & VLA & $0.64 " \times 0.33^{\prime \prime}$ & 5.0 & 75 \\
\hline $22 \mathrm{MHz} \mathrm{H}_{2} \mathrm{O}$ maser & 180614.9 & -203141 & VLA & $0.64 " \times 0.33^{\prime \prime}$ & 6.0 & 9.5 \\
\hline $22 \mathrm{MHz} \mathrm{H}_{2} \mathrm{O}$ maser & 180615.0 & -203142 & VLA & $0.64 " \times 0.33^{\prime \prime}$ & 25 & 0.30 \\
\hline 8.2 GHz and $9.2 \mathrm{GHz}$ UCHII region ${ }^{\mathrm{f}}$ & 180614.7 & -203132 & ATCA $^{\mathrm{g}}$ & $1.1^{\prime \prime} \times 1.2^{\prime \prime}$ & $\ldots^{\mathrm{h}}$ & $\ldots^{\mathrm{h}}$ \\
\hline 8.2 GHz and $9.2 \mathrm{GHz}$ UCHII region & 180614.4 & -203126 & ATCA & $2.2^{\prime \prime} \times 1.6^{\prime \prime}$ & $\ldots$ & $\ldots$ \\
\hline 8.2 GHz and $9.2 \mathrm{GHz}$ UCHII region & 180614.9 & -203143 & ATCA & $0.7^{\prime \prime} \times 0.6^{\prime \prime}$ & $\ldots$ & $\ldots$ \\
\hline
\end{tabular}

${ }^{\mathrm{a}}$ All $44 \mathrm{GHz}$ class I methanol masers in this section were found in [11] and [13]; ${ }^{\mathrm{b}}$ The Karl G. Jansky Very Large Array. ${ }^{\mathrm{c}}$ 25]; ${ }^{\mathrm{d}}$ The Very Long Baseline Array. ${ }^{\mathrm{I}}$ Information on all water masers taken from [18] unless otherwise noted. ${ }^{\mathrm{f}}$ All of these UC H II regions were reported in [24]. [24] explain that their observations were taken at both 8.2 and $9.2 \mathrm{GHz} .{ }^{\mathrm{g}}$ The Australia Telescope Compact Array (ATCA). ${ }^{\mathrm{h}} \mathrm{UC}$ HII regions are continuum data and, therefore, produce no line profile information. Note: Sources consulted for data presented in tables one through eight reported different significant figures. For convenience the data have been rounded to the least number of significant figures. Information on the resolution of each observing instrument is presented in the column "synthesized beam" which differs between instruments and configurations of the same instrument.



Figure 4. Plot of intensities and center velocities $\left(v_{\mathrm{LSR}}\right)$ of methanol and $\mathrm{H}_{2} \mathrm{O}$ masers associated with G9.62 +0.19 . At the top of each line is a symbol indicating the type of source; the Class I methanol maser is represented by a diamond, the Class II methanol maser by a triangle, and the $\mathrm{H}_{2} \mathrm{O}$ masers by filled circles. A $\mathrm{H}_{2} \mathrm{O}$ maser with a center velocity of $25 \mathrm{~km} \cdot \mathrm{s}^{-1}$ is outside the range of this plot. In this, and all other such figures, the $v_{\mathrm{LSR}}$ of the Class II methanol maser will be used as a reference; masers with $v_{\mathrm{LSR}}$ larger than the Class II methanol maser will be considered to be redshifted, and masers with $v_{\mathrm{LSR}}$ smaller than the Class II methanol maser will be considered to be blueshifted. 


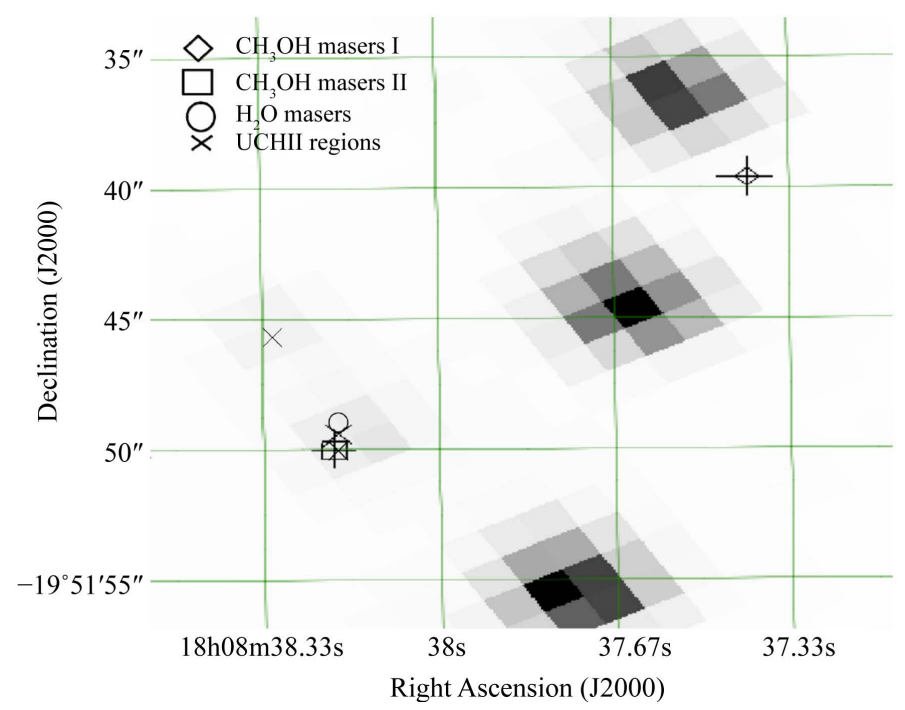

Figure 5. Figure showing the Class II and the Class I methanol maser and other sources of interest in region G10.47 +0.03 . The symbols are the same as in Figure 3, and are also marked in a box at the top left of the figure. The grayscale shows the $4.5 \mu \mathrm{m}$ Spitzer infrared image and the intensity range is between $2.22 \mathrm{MJy} \cdot \mathrm{sr}^{-1}$ and $1100 \mathrm{MJy} \cdot \mathrm{sr}^{-1}$.

position is marked by a square in Figure 5. The Class I and Class II masers are separated by about 15.3"; at a distance of $6.0 \mathrm{kpc}$ to G10.47 + 0.03 ([14]), this is equivalent to about 0.44 pc. Two of the $\mathrm{H}_{2} \mathrm{O}$ masers in this source are almost coincident with the Class II methanol maser, and a third is right next to it. There are three UC $\mathrm{H}$ II regions, two of which are almost coincident with the Class II methanol maser, and a third lies to the northeast of it. A line of strong $4.5 \mu \mathrm{m}$ point sources lies to the east and southeast of the Class I methanol maser. The positions of these sources, together with the telescopes used to observe them and the angular resolution in terms of synthesized beam of the observations, are listed in Table 2.

The center velocities $\left(v_{\mathrm{LSR}}\right)$ and intensities of the Class I and Class II methanol masers and $\mathrm{H}_{2} \mathrm{O}$ masers have been plotted in Figure 6. All the three $\mathrm{H}_{2} \mathrm{O}$ masers shown in this figure are at smaller $v_{\mathrm{LSR}}$ (i.e., blueshifted) than the Class II methanol maser. The Class I methanol maser is also blueshifted with respect to the Class II methanol maser.

In a previous version, the label on this region was a typo. It was G10.47 + 0.27 when I clearly meant G10.47 + 0.03 .

\subsection{Region G12.20 - 0.11}

Figure 7 shows the Class II methanol maser and the Class I methanol maser in G12.20 - 0.11, together with other sources of interest. The Class I and Class II masers are separated by about 32.4"; at a distance of $8.3 \mathrm{kpc}$ to G12.20 - 0.11 ([14]), this is equivalent to about 1.3 pc. Six of the $\mathrm{H}_{2} \mathrm{O}$ masers in this source are clustered around the Class I methanol maser. An ultracompact HII region is to the south of the Class I methanol maser. To the east of the Class I methanol maser there is a strong $4.5 \mu \mathrm{m}$ infrared source. Figure 8 shows the line velocities of the masers in this region. The positions of these sources, together with the telescopes used to observe them and the angular resolution (in terms of synthesized beam) of the observations, are listed in Table 3.

The center velocities $\left(v_{\mathrm{LSR}}\right)$ and intensities of the Class I and Class II methanol masers and $\mathrm{H}_{2} \mathrm{O}$ masers have been plotted in Figure 8. Four of the eight $\mathrm{H}_{2} \mathrm{O}$ masers are at $v_{\mathrm{LSR}}$ greater than (i.e., redshifted) the $v_{\mathrm{LSR}}$ of the Class II methanol maser. The Class I methanol maser is also redshifted with respect to the Class II methanol maser.

\subsection{Region G31.41 + 0.31}

Figure 9 shows the Class II methanol maser and the Class I methanol maser in G31.41 + 0.31, together with 
Table 2. Positions and associated information for sources associated with G10.47 + 0.03.

\begin{tabular}{|c|c|c|c|c|c|c|}
\hline Source type & R.A. (J2000) & Dec. (J2000) & Observing & Synthesized & Kinematic & \\
\hline & (h m s) & $\left(0^{\prime}+"\right)$ & Telescope & Beam & $v_{\mathrm{LSR}}\left(\mathrm{km} \cdot \mathrm{s}^{-1}\right)$ & Intensity (Jy) \\
\hline $44 \mathrm{GHz}$ Class $\mathrm{I} \mathrm{CH}_{3} \mathrm{OH}$ maser ${ }^{\mathrm{a}}$ & 180837.4 & -195140 & VLA & $3.14 " \times 1.33^{\prime \prime}$ & 67 & 1.9 \\
\hline 6.7 GHz Class II $\mathrm{CH}_{3} \mathrm{OH}$ maser & 180838.2 & -195150 & ATCA & $1.5^{\prime \prime} \times 1.5^{\prime \prime}$ & 75 & 120 \\
\hline $22 \mathrm{GHz} \mathrm{H}_{2} \mathrm{O}$ maser $^{\mathrm{c}}$ & 180838.2 & -195150 & VLA & $0.48 " \times 0.45^{\prime \prime}$ & 64 & 200 \\
\hline $22 \mathrm{GHz} \mathrm{H}_{2} \mathrm{O}$ maser & 180838.2 & -195150 & VLA & $0.48 " \times 0.45^{\prime \prime}$ & 63 & 14 \\
\hline $22 \mathrm{GHz} \mathrm{H}_{2} \mathrm{O}$ maser & 180838.2 & -195149 & VLA & $0.48 " \times 0.45^{\prime \prime}$ & 70 & 28 \\
\hline 14.9 GHz UCHII region ${ }^{\mathrm{d}}$ & 180838.2 & -195150 & VLA & $0.89 " \times 0.57^{\prime \prime}$ & $\ldots$ & $\ldots$ \\
\hline 14.9 GHz UCHII region & 180838.2 & -195149 & VLA & $0.79^{\prime \prime} \times 0.56^{\prime \prime}$ & $\ldots$ & $\ldots$ \\
\hline 14.9 GHz UCHII region & 180838.3 & -195145 & VLA & $1.04 " \times 0.79 "$ & $\ldots$ & $\ldots$ \\
\hline
\end{tabular}

${ }^{\mathrm{a}}[13] ;{ }^{\mathrm{b}}[26] ;{ }^{\mathrm{c}}[18] ;{ }^{\mathrm{d}}[19]$.

Table 3. Positions and associated information for sources associated with G12.20 - 0.11.

\begin{tabular}{|c|c|c|c|c|c|c|}
\hline Source type & R.A. (J2000) & Dec. (J2000) & Observing & Synthesized & Kinematic & \\
\hline & (h m s) & $\left({ }^{\prime}+"\right)$ & Telescope & Beam & $v_{\mathrm{LSR}}\left(\mathrm{km} \cdot \mathrm{s}^{-1}\right)$ & Intensity (Jy) \\
\hline $44 \mathrm{GHz}$ Class I CH $\mathrm{CH}_{3} \mathrm{OH}$ maser $^{\mathrm{a}}$ & 181239.9 & -182415 & VLA & $2.93^{\prime \prime} \times 1.43^{\prime \prime}$ & 24 & 4.3 \\
\hline $6.7 \mathrm{GHz}$ Class II $\mathrm{CH}_{3} \mathrm{OH}$ maser ${ }^{\mathrm{b}}$ & 181240.1 & -182447 & ATCA & $1.5^{\prime \prime} \times 1.5^{\prime \prime}$ & 20 & 15 \\
\hline $22 \mathrm{GHz} \mathrm{H}_{2} \mathrm{O}$ maser $^{\mathrm{c}}$ & 181239.9 & -182417 & VLA & $0.49^{\prime \prime} \times 0.38^{\prime \prime}$ & 20 & 120 \\
\hline $22 \mathrm{GHz} \mathrm{H}_{2} \mathrm{O}$ maser & 181239.8 & -182417 & VLA & $0.49^{\prime \prime} \times 0.38 "$ & -4.5 & 110 \\
\hline $22 \mathrm{GHz} \mathrm{H}_{2} \mathrm{O}$ maser & 181244.5 & -182425 & VLA & $0.49^{\prime \prime} \times 0.38 "$ & 26 & 70 \\
\hline $22 \mathrm{GHz} \mathrm{H}_{2} \mathrm{O}$ maser & 181239.8 & -182418 & VLA & $0.49^{\prime \prime} \times 0.38 "$ & 23 & 3.2 \\
\hline $22 \mathrm{GHz} \mathrm{H}_{2} \mathrm{O}$ maser & 181239.8 & -182416 & VLA & $0.49^{\prime \prime} \times 0.38 "$ & 32 & 1.5 \\
\hline $22 \mathrm{GHz} \mathrm{H}_{2} \mathrm{O}$ maser & 181244.5 & -182424 & VLA & $0.49^{\prime \prime} \times 0.38 "$ & 28 & 2.3 \\
\hline $22 \mathrm{GHz} \mathrm{H}_{2} \mathrm{O}$ maser & 181239.9 & -182416 & VLA & $0.49^{\prime \prime} \times 0.38 "$ & 8.0 & 0.50 \\
\hline $22 \mathrm{GHz} \mathrm{H}_{2} \mathrm{O}$ maser & 181240.1 & -182416 & VLA & $0.49^{\prime \prime} \times 0.38^{\prime \prime}$ & -18 & 0.40 \\
\hline 14.9 GHz UCHII region ${ }^{d}$ & 181238.1 & -182510 & VLA & $0.4^{\prime \prime} \times 0.4 "$ & $\ldots$ & $\ldots$ \\
\hline 14.9 GHz UCHII region & 181243.7 & -182544 & VLA & $0.4^{\prime \prime} \times 0.4^{\prime \prime}$ & $\ldots$ & $\ldots$ \\
\hline 14.9 GHz UCHII region & 181239.9 & -182426 & VLA & $0.4^{\prime \prime} \times 0.4^{\prime \prime}$ & $\ldots$ & $\ldots$ \\
\hline
\end{tabular}

a $[13] ;{ }^{\mathrm{b}}[26] ;{ }^{\mathrm{c}}[18] ;{ }^{\mathrm{d}}[19]$.

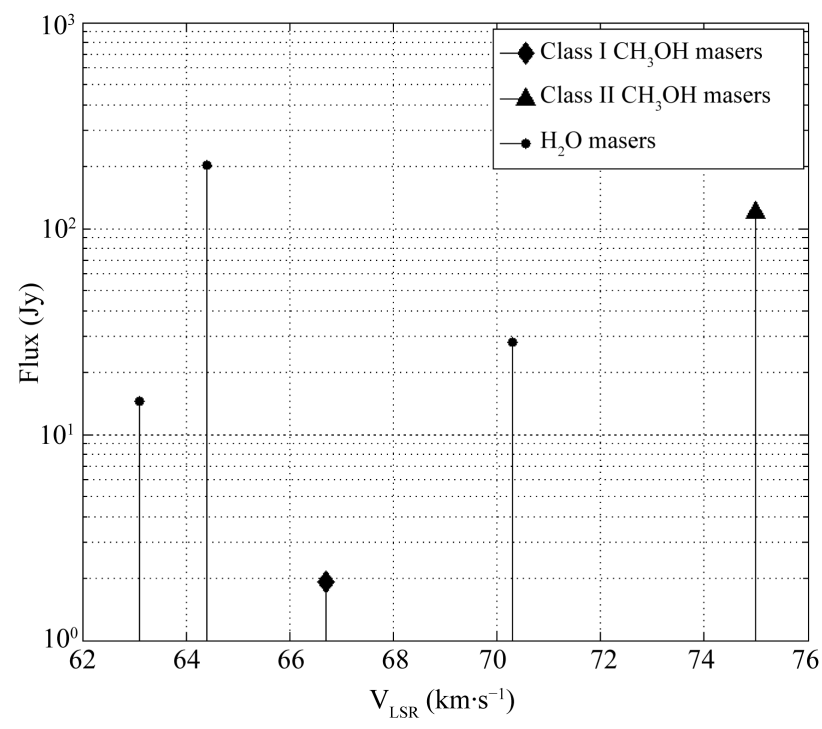

Figure 6. Plot of intensities and center velocities $\left(v_{\mathrm{LSR}}\right)$ of methanol and $\mathrm{H}_{2} \mathrm{O}$ masers associated with $\mathrm{G} 10.47+0.03$. The symbols are the same as in Figure 4. 


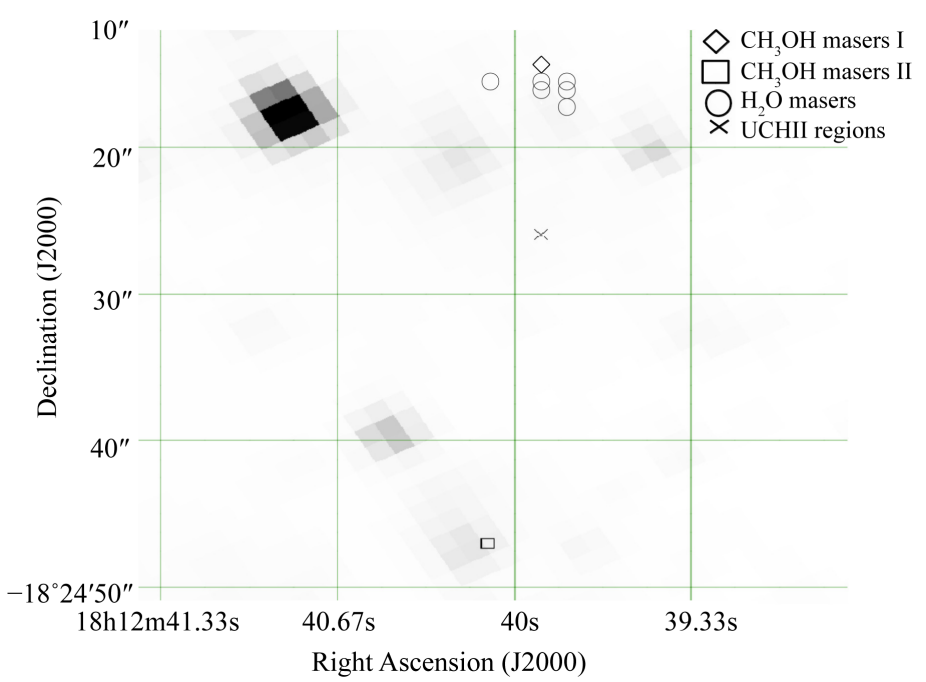

Figure 7. Figure showing the Class II and the Class I methanol maser and other sources of interest in region G12.20 - 0.11. The grayscale shows the $4.5 \mu \mathrm{m}$ Spitzer infrared image and the intensity range is between $2.58 \mathrm{MJy} \cdot \mathrm{sr}^{-1}$ and $1220 \mathrm{MJy} \cdot \mathrm{sr}^{-1}$. The symbols are the same as in Figure 3, and are also marked in a box at the top right of the figure.

other sources of interest. The Class I methanol maser lies about 20.43" to the southeast of the Class I methanol maser; at a distance of $7.3 \mathrm{kpc}$ to G31.41 + 0.31 ([14]), this is equivalent to about $0.72 \mathrm{pc}$. A complex of $\mathrm{H}_{2} \mathrm{O}$ masers is about 5" north of the Class I methanol maser position. A UC HII region is 7" to the northeast of the Class I methanol maser. This region shows two weak $4.5 \mu \mathrm{m}$ infrared sources, one about 10" north of the Class I methanol maser and the other to the west of the Class II methanol maser. The positions of these sources, together with the telescopes used to observe them and the angular resolution (in terms of synthesized beam) of the observations, are listed in Table 4.

The center velocities $\left(v_{\mathrm{LSR}}\right)$ and intensities of the Class I and Class II methanol masers and $\mathrm{H}_{2} \mathrm{O}$ masers have been plotted in Figure 10. Six of the eight $\mathrm{H}_{2} \mathrm{O}$ masers shown in this figure are at lower $v_{\mathrm{LSR}}$ (i.e., blueshifted) than the Class II methanol maser. The Class I methanol maser is also blueshifted with respect to the Class II methanol maser.

\subsection{Region G35.03 + 0.35}

Figure 11 shows the Class II methanol maser and the Class I methanol maser in G35.03 + 0.35, together with other sources of interest. The Class I methanol maser lies almost due west of the Class II methanol maser by about 11.98"; at a distance of $6.9 \mathrm{kpc}$ to G35.03 + 0.35 ([14]), this is equivalent to about $0.40 \mathrm{pc}$. There is only one $\mathrm{H}_{2} \mathrm{O}$ maser in this source, and it lies to the west of the Class I methanol maser. Extended $4.5 \mu \mathrm{m}$ emission straddles the region between the Class I methanol and $\mathrm{H}_{2} \mathrm{O}$ maser. The positions of these sources, together with the telescopes used to observe them and the angular resolution (in terms of synthesized beam) of the observations, are listed in Table 5 .

The center velocities $\left(v_{\mathrm{LSR}}\right)$ and intensities of the Class I and Class II methanol masers and $\mathrm{H}_{2} \mathrm{O}$ masers have been plotted in Figure 12. The $\mathrm{H}_{2} \mathrm{O}$ maser is at a higher $v_{\mathrm{LSR}}$ (i.e., redshifted) than the Class II methanol maser. The Class I methanol maser is also redshifted with respect to the Class II methanol maser.

\subsection{Region G45.07 + 0.13}

Figure 13 shows the Class II methanol maser and the Class I methanol maser in G45.07 + 0.13, together with other sources of interest. To the south of the Class I maser position is a Class II methanol maser, whose position is marked by a square in Figure 13. The Class I and Class II masers are separated by about 5.96"; at a distance of $6.0 \mathrm{kpc}$ to G45.07 + 0.13 ([14]), this is equivalent to about 0.17 pc. A UC HII region is almost coincident with the Class II methanol maser position, and there is $4.5 \mu \mathrm{m}$ emission in the entire region. The positions of 

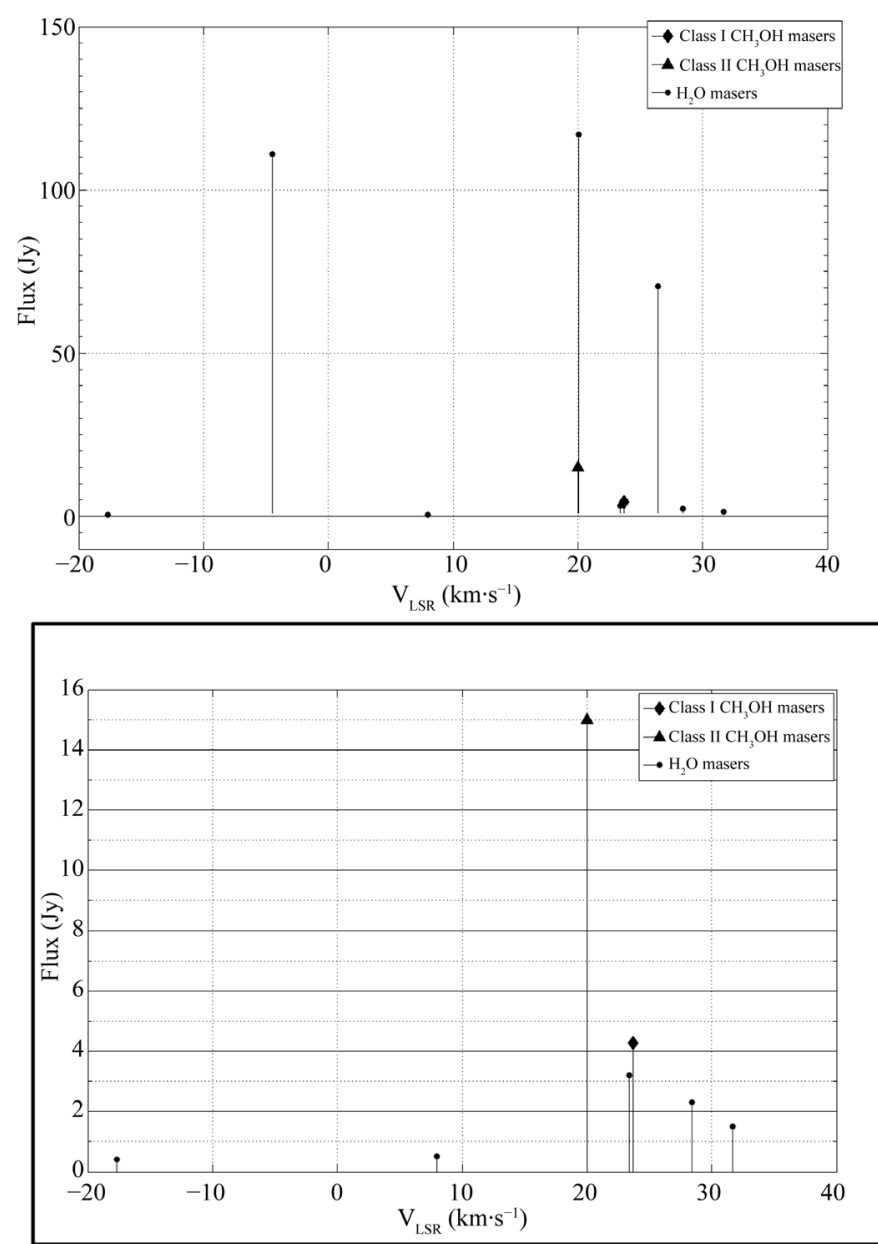

Figure 8. Plot of intensities and center velocities $\left(v_{\mathrm{LSR}}\right)$ of methanol and $\mathrm{H}_{2} \mathrm{O}$ masers associated with G12.20 - 0.11. The symbols are the same as in Figure 4. For better visibility, an enlarged version of the $0-16 \mathrm{Jy}$ segment in the upper plot is presented in the lower plot.

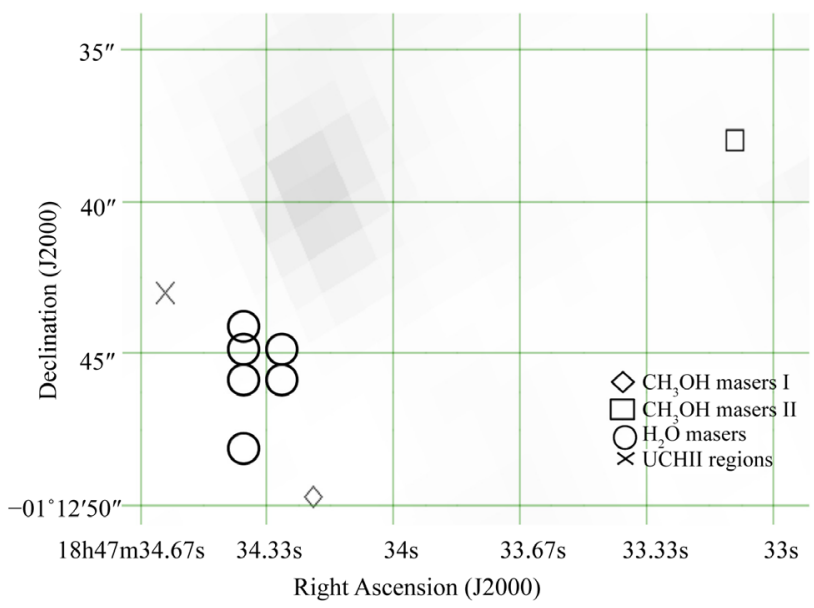

Figure 9. Figure showing the Class II and the Class I methanol maser and other sources of interest in region G31.41 + 0.31. The grayscale shows the $4.5 \mu \mathrm{m}$ Spitzer infrared image and the intensity range is between 1.13 $\mathrm{MJy} \cdot \mathrm{Sr}^{-1}$ and $658 \mathrm{MJy} \cdot \mathrm{sr}^{-1}$. The symbols are the same as in Figure 3. 
Table 4. Positions and associated information for sources associated with G31.41 + 0.31.

\begin{tabular}{|c|c|c|c|c|c|c|}
\hline Source type & R.A. (J2000) & Dec. (J2000) & Observing & Synthesized & Kinematic & \\
\hline & (h m s) & $\left({ }^{\circ}+"\right)$ & Telescope & Beam & $v_{\mathrm{LSR}}\left(\mathrm{km} \cdot \mathrm{s}^{-1}\right)$ & Intensity (Jy) \\
\hline $44 \mathrm{GHz}$ Class I $\mathrm{CH}_{3} \mathrm{OH}$ maser $^{\mathrm{a}}$ & 184734.2 & -011250 & VLA & $2.21 " \times 1.36^{\prime \prime}$ & 100 & 1.7 \\
\hline 6.7 GHz Class II $\mathrm{CH}_{3} \mathrm{OH}$ maser ${ }^{\mathrm{b}}$ & 184733.1 & -011238 & ATCA & $1.5^{\prime \prime} \times 1.5^{\prime \prime}$ & 104 & 11 \\
\hline $22 \mathrm{GHz} \mathrm{H}_{2} \mathrm{O}$ maser $^{\mathrm{c}}$ & 184734.3 & -011245 & VLA & $0.47^{\prime \prime} \times 0.47 "$ & 104 & 130 \\
\hline $22 \mathrm{GHz} \mathrm{H}_{2} \mathrm{O}$ maser & 184734.3 & -011246 & VLA & $0.47^{\prime \prime} \times 0.47^{\prime \prime}$ & 103 & 11 \\
\hline $22 \mathrm{GHz} \mathrm{H}_{2} \mathrm{O}$ maser & 184734.3 & -011246 & VLA & $0.47 " \times 0.47^{\prime \prime}$ & 95.3 & 16 \\
\hline $22 \mathrm{GHz} \mathrm{H}_{2} \mathrm{O}$ maser & 184734.3 & -011246 & VLA & $0.47^{\prime \prime} \times 0.47^{\prime \prime}$ & 96.3 & 35 \\
\hline $22 \mathrm{GHz} \mathrm{H}_{2} \mathrm{O}$ maser & 184734.4 & -011244 & VLA & $0.47^{\prime \prime} \times 0.47^{\prime \prime}$ & 117 & 2.6 \\
\hline $22 \mathrm{GHz} \mathrm{H}_{2} \mathrm{O}$ maser & 184734.4 & -011245 & VLA & $0.47^{\prime \prime} \times 0.47^{\prime \prime}$ & 99.3 & 44 \\
\hline $22 \mathrm{GHz} \mathrm{H}_{2} \mathrm{O}$ maser & 184734.4 & -011246 & VLA & $0.47^{\prime \prime} \times 0.47^{\prime \prime}$ & 97.6 & 11 \\
\hline $22 \mathrm{GHz} \mathrm{H}{ }_{2} \mathrm{O}$ maser & 184734.4 & -011248 & VLA & $0.47^{\prime \prime} \times 0.47^{\prime \prime}$ & 124 & 0.40 \\
\hline 14.9 GHz UCHII region ${ }^{\mathrm{d}}$ & 184734.6 & -011243 & VLA & $1.1^{\prime \prime} \times 1.1^{\prime \prime}$ & $\ldots$ & $\ldots$ \\
\hline
\end{tabular}

${ }^{\mathrm{a}}[13] ;{ }^{\mathrm{b}}[26] ;{ }^{\mathrm{c}}[18] ;{ }^{\mathrm{d}}[19]$.
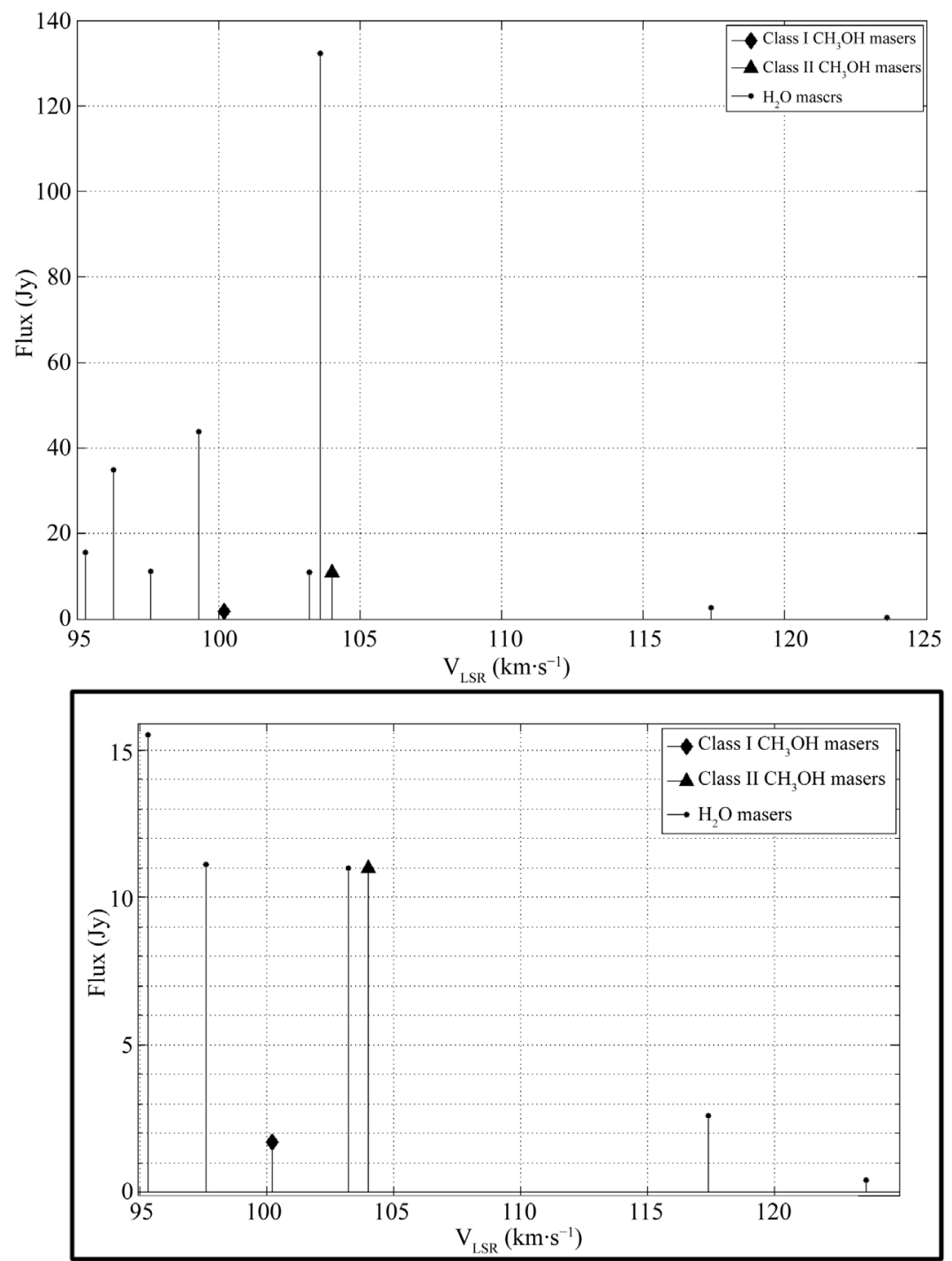

Figure 10. Plot of intensities and center velocities $\left(v_{\mathrm{LSR}}\right)$ of methanol and $\mathrm{H}_{2} \mathrm{O}$ masers associated with G31.41 + 0.31. The symbols are the same as in Figure 4. The lower plot is an enlarged version of the $0-15$ Jy segment in the upper plot. 


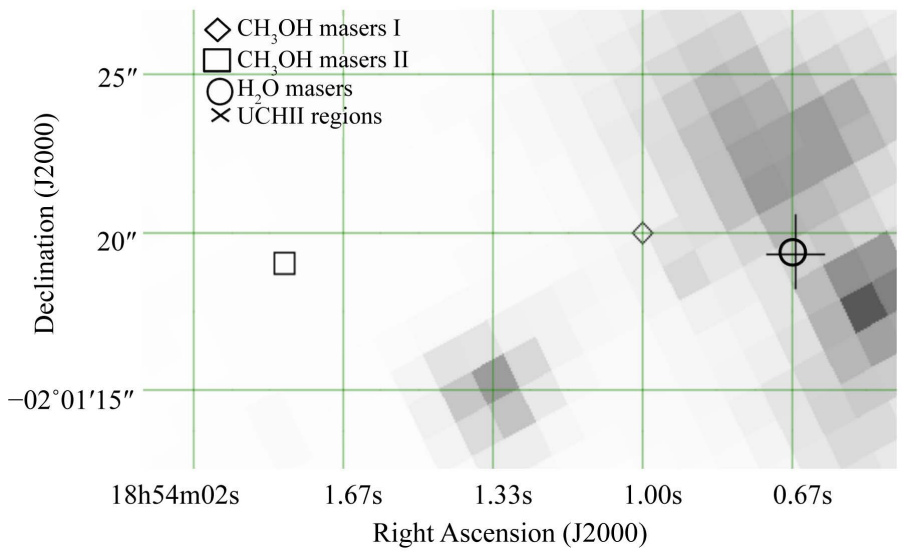

Figure 11. Figure showing the Class II and the Class I methanol maser and other sources of interest in region G35.03 + 0.35. The grayscale shows the $4.5 \mu \mathrm{m}$ Spitzer infrared image and the intensity range is between 0.374 $\mathrm{MJy} \cdot \mathrm{Sr}^{-1}$ and $455 \mathrm{MJy} \cdot \mathrm{sr}^{-1}$. The symbols are the same as in Figure 3.
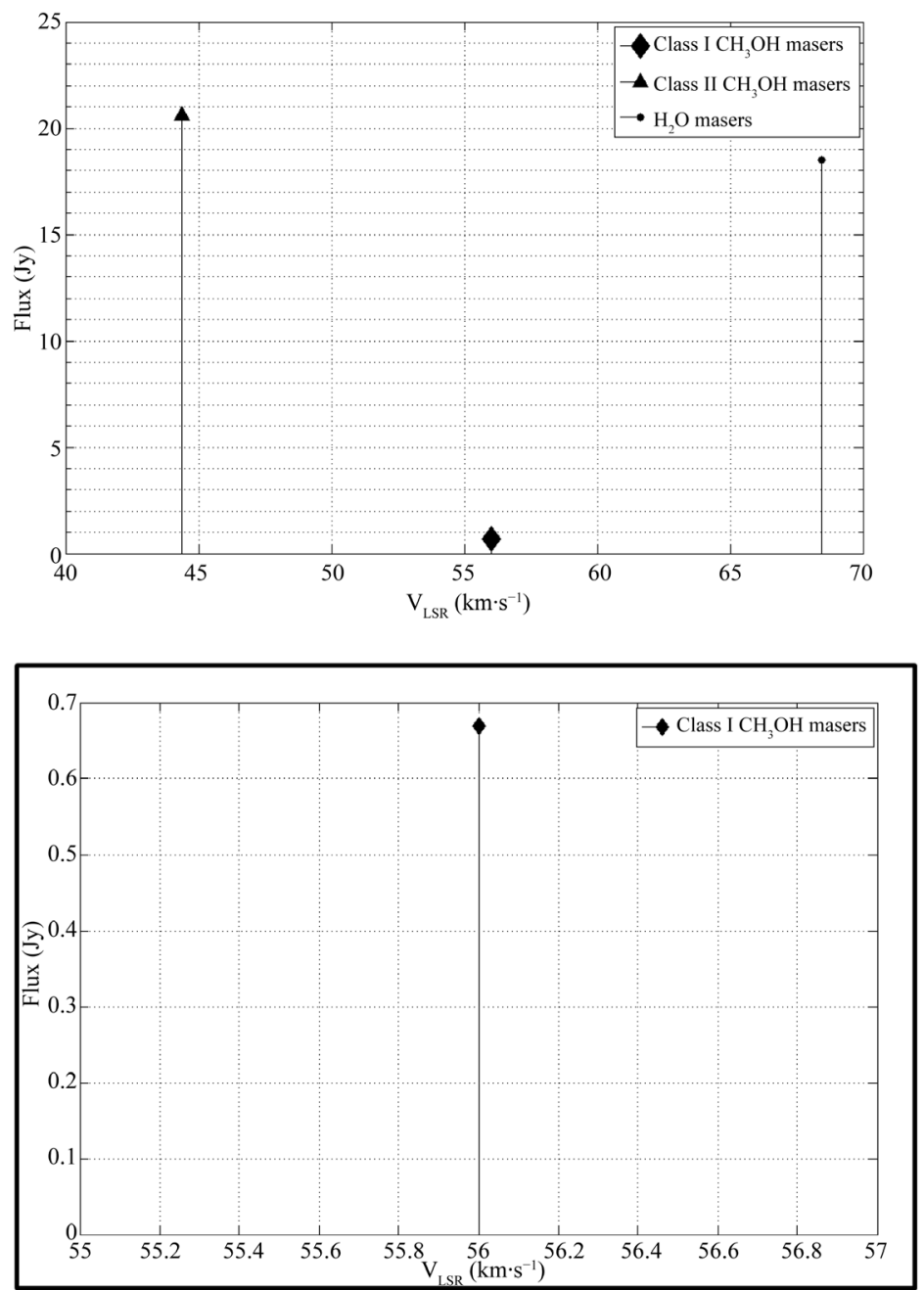

Figure 12. Plot of intensities and center velocities $\left(v_{\mathrm{LSR}}\right)$ of methanol and $\mathrm{H}_{2} \mathrm{O}$ masers associated with G35.03 + 0.35. The symbols are the same as in Figure 4 . The lower plot is an enlarged version of the $0-0.7 \mathrm{Jy}$ segment in the upper plot. 
Table 5. Positions and associated information for sources associated with G35.03 + 0.35.

\begin{tabular}{|c|c|c|c|c|c|c|}
\hline Source type & R.A. (J2000) & Dec. (J2000) & Observing & Synthesized & Kinematic & \\
\hline & (h m s) & $\left({ }^{\circ}+"\right)$ & Telescope & Beam & $v_{\mathrm{LSR}}\left(\mathrm{km} \cdot \mathrm{s}^{-1}\right)$ & Intensity (Jy) \\
\hline $44 \mathrm{GHz}$ Class $\mathrm{I} \mathrm{CH}_{3} \mathrm{OH}$ maser $^{\mathrm{a}}$ & 18541.0 & +020120 & VLA & $0.58^{\prime \prime} \times 0.46^{\prime \prime}$ & 56 & 0.67 \\
\hline $6.7 \mathrm{GHz}$ Class II $\mathrm{CH}_{3} \mathrm{OH}$ maser & 18541.8 & +020119 & VLA & $2.32 "$ × 1.34" & 44 & 21 \\
\hline $22 \mathrm{GHz} \mathrm{H}_{2} \mathrm{O}$ maser $^{\mathrm{c}}$ & 18540.66 & +020119 & VLA & $2.6^{\prime \prime} \times 1.8^{\prime \prime}$ & 68 & 19 \\
\hline 14.9 GHz UCHII region ${ }^{\mathrm{d}}$ & 185129 & +015731 & VLA & $0.93^{\prime \prime} \times 0.8 "$ & $\ldots$ & $\ldots$ \\
\hline
\end{tabular}

${ }^{\mathrm{a}}[13] ;{ }^{\mathrm{b}}[26] ;{ }^{\mathrm{c}}[18] ;{ }^{\mathrm{d}}[19]$.

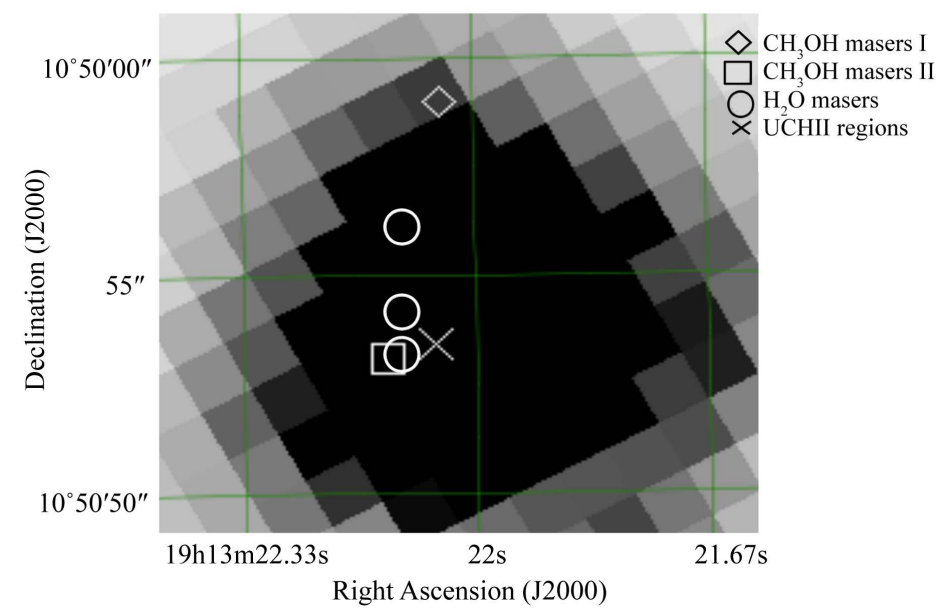

Figure 13. Figure showing the Class II and the Class I methanol maser and other sources of interest in region $\mathrm{G} 45.07+0.13$. The grayscale shows the $4.5 \mu \mathrm{m}$ Spitzer infrared image and the intensity range is between 0.0683 $\mathrm{MJy} \cdot \mathrm{sr}^{-1}$ and $543 \mathrm{MJy} \cdot \mathrm{sr}^{-1}$. The symbols are the same as in Figure 3.

these sources, together with the telescopes used to observe them and the angular resolution (in terms of synthesized beam) of the observations, are listed in Table 6 .

The center velocities $\left(v_{\mathrm{LSR}}\right)$ and intensities of the Class I and Class II methanol masers and $\mathrm{H}_{2} \mathrm{O}$ masers have been plotted in Figure 14. Four of the five $\mathrm{H}_{2} \mathrm{O}$ masers shown in this figure are at larger $v_{\text {LSR }}$ (i.e., redshifted) than the Class II methanol maser. The Class I methanol maser is also redshifted with respect to the Class II methanol maser.

\subsection{Region G45.47 + 0.07}

Figure 15 shows the Class II methanol maser and the Class I methanol maser in G45.47 + 0.07, together with other sources of interest. The Class I and Class II masers are separated by about 115.2"; at a distance of $4.0 \mathrm{kpc}$ to G45.47 + 0.07 ([14]), this is equivalent to about 2.23 pc. A UC HII region is 12" due south of the Class I methanol maser position. The $4.5 \mu \mathrm{m}$ infrared emission is present throughout the region, with discrete enhancements at several locations, including one that is almost coincident with the position of the UC HII region. The positions of these sources, together with the telescopes used to observe them and the angular resolution (in terms of synthesized beam) of the observations, are listed in Table 7.

The center velocities ( $\left.v_{\mathrm{LSR}}\right)$ and intensities of the Class I and Class II methanol masers and $\mathrm{H}_{2} \mathrm{O}$ masers have been plotted in Figure 16. Both of the $\mathrm{H}_{2} \mathrm{O}$ masers are at larger $v_{\mathrm{LSR}}$ (i.e., redshifted) than the Class II methanol maser. The Class I methanol maser is also redshifted with respect to the the Class II methanol maser.

\subsection{Region G75.78 + 0.34}

Figure 17 shows the Class II methanol maser and the Class I methanol maser in G75.78 + 0.34, together with other sources of interest. The Class I and Class II masers are separated by about 7.12"; at a distance of 4.9 kpc to 
Table 6. Positions and associated information for sources associated with G45.07 + 0.13.

\begin{tabular}{|c|c|c|c|c|c|c|}
\hline Source type & R.A. (J2000) & Dec. (J2000) & Observing & Synthesized & Kinematic & \\
\hline & (h m s) & $\left({ }^{\circ} \cdot "\right)$ & Telescope & Beam & $v_{\mathrm{LSR}}\left(\mathrm{km} \cdot \mathrm{s}^{-1}\right)$ & Intensity (Jy) \\
\hline $44 \mathrm{GHz}$ Class I $\mathrm{CH}_{3} \mathrm{OH}$ maser $^{\mathrm{a}}$ & 191322.1 & +105059 & VLA & $1.98 "$ × 1.6" & 59.3 & 1.1 \\
\hline $6.7 \mathrm{GHz}$ Class II $\mathrm{CH}_{3} \mathrm{OH}$ maser ${ }^{\mathrm{b}}$ & 191322.1 & +105053 & MERLIN $^{\mathrm{e}}$ & $0.4^{\prime \prime} \times 0.4^{\prime \prime}$ & 57.8 & 64 \\
\hline $22 \mathrm{GHz} \mathrm{H}_{2} \mathrm{O}$ maser $^{\mathrm{c}}$ & 191322.1 & +105053 & VLA & $0.61 " \times 0.37 "$ & 60.7 & 11 \\
\hline 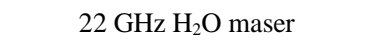 & 191322.1 & +105053 & VLA & $0.61 " \times 0.37 "$ & 51.8 & 2.8 \\
\hline $22 \mathrm{GHz} \mathrm{H}_{2} \mathrm{O}$ maser & 191322.1 & +105054 & VLA & $2.6 " \times 0.37 "$ & 63.3 & 45 \\
\hline $22 \mathrm{GHz} \mathrm{H}_{2} \mathrm{O}$ maser & 191322.1 & +105056 & VLA & $0.4 " \times 0.37 "$ & 60.6 & 22 \\
\hline $22 \mathrm{GHz} \mathrm{H}_{2} \mathrm{O}$ maser & 191322.1 & +105056 & VLA & $0.4 " \times 0.37 "$ & 64.3 & 38 \\
\hline 14.9 GHz UCHII region ${ }^{\mathrm{d}}$ & 191323.7 & +105052 & VLA & $0.80^{\prime \prime} \times 0.72^{\prime \prime}$ & $\cdots$ & $\cdots$ \\
\hline
\end{tabular}

a[13]; ${ }^{\mathrm{b}}[26] ;{ }^{\mathrm{c}}[18]$; ${ }^{\mathrm{d}}[19] . ;{ }^{\mathrm{e}}$ Multi-Element Radio Linked Interferometer Network (MERLIN).
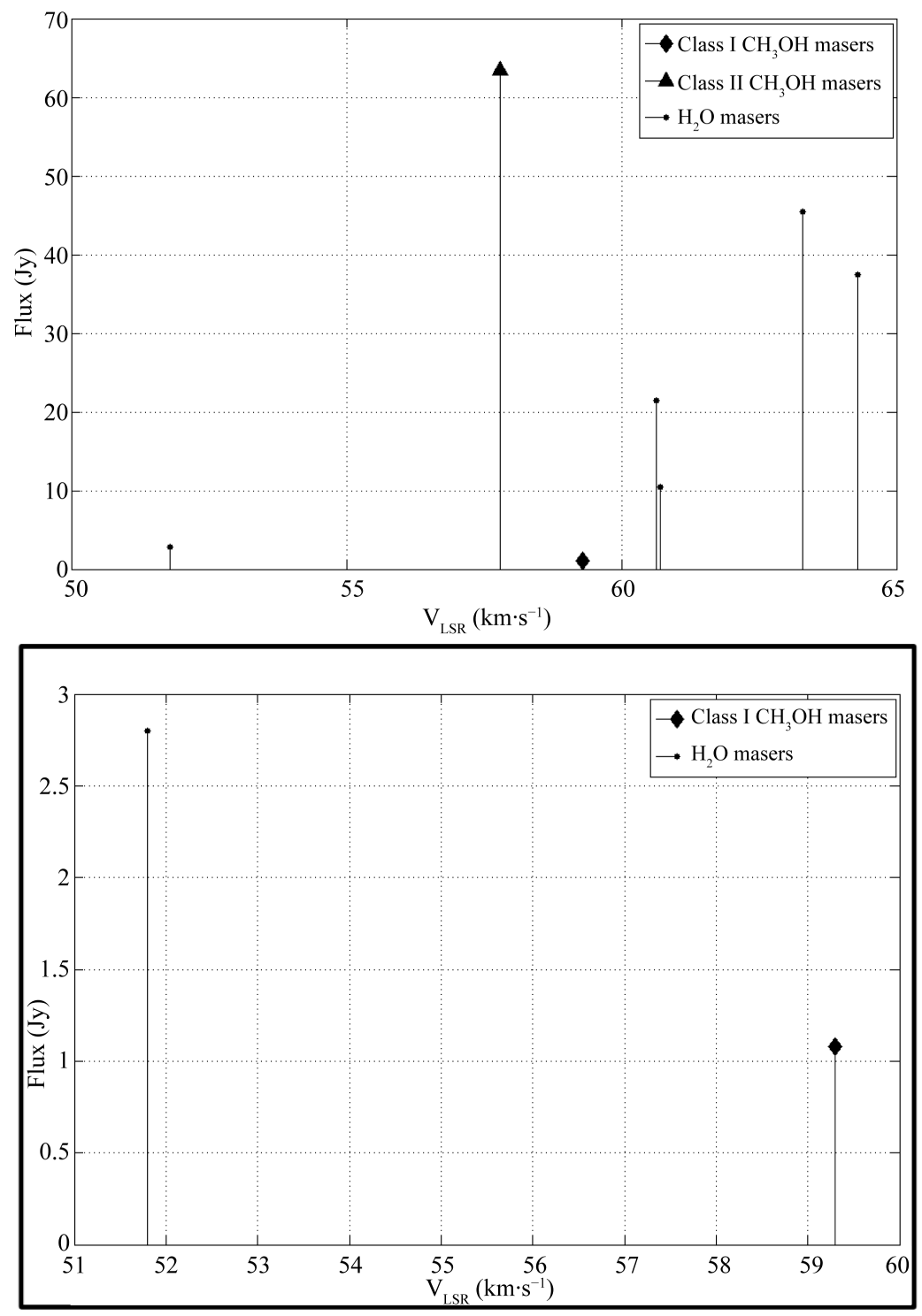

Figure 14. Plot of intensities and center velocities $\left(v_{\mathrm{LSR}}\right)$ of methanol and $\mathrm{H}_{2} \mathrm{O}$ masers associated with G45.07 + 0.13. The symbols are the same as in Figure 4. The lower plot is an enlarged version of the $0-3$ Jy segment in the upper plot. 
Table 7. Positions and associated information for sources associated with G45.47 + 0.07.

\begin{tabular}{|c|c|c|c|c|c|c|}
\hline Source type & R.A. (J2000) & Dec. (J2000) & Observing & Synthesized & Kinematic & \\
\hline & (h m s) & $\left({ }^{\circ} \cdot "\right)$ & Telescope & Beam & $v_{\mathrm{LSR}}\left(\mathrm{km} \cdot \mathrm{s}^{-1}\right)$ & Intensity (Jy) \\
\hline $44 \mathrm{GHz}$ Class I CH $\mathrm{CH}_{3} \mathrm{OH}$ maser ${ }^{\mathrm{a}}$ & 191425.7 & +110937 & VLA & $2.17^{\prime \prime} \times 1.54^{\prime \prime}$ & 65 & 0.55 \\
\hline $6.7 \mathrm{GHz}$ Class II $\mathrm{CH}_{3} \mathrm{OH}$ maser & 191418.3 & +110859 & MERLIN & $0.1^{\prime \prime} \times 0.1^{\prime \prime}$ & 50 & 0.68 \\
\hline $22 \mathrm{GHz} \mathrm{H}_{2} \mathrm{O}$ maser $^{\mathrm{c}}$ & 191418.1 & +110847 & VLA & $2.6^{\prime \prime} \times 1.8^{\prime \prime}$ & 61 & 4.4 \\
\hline $22 \mathrm{GHz} \mathrm{H}_{2} \mathrm{O}$ maser & 191425.7 & +110930 & VLA & $3.5^{\prime \prime} \times 1.1^{\prime \prime}$ & 69 & 1.3 \\
\hline 5 GHz UCHII region ${ }^{\mathrm{d}}$ & 191425.7 & +110926 & VLA & $0.42^{\prime \prime} \times 0.42^{\prime \prime}$ & $\ldots$ & $\ldots$ \\
\hline 14.9 GHz UCHII region ${ }^{\mathrm{e}}$ & 191419.6 & +111035 & VLA & $0.4^{\prime \prime} \times 0.4^{\prime \prime}$ & $\ldots$ & $\ldots$ \\
\hline
\end{tabular}

${ }^{\mathrm{a}}[13] ;{ }^{\mathrm{b}}[16] ;{ }^{\mathrm{c}}[18] ;{ }^{\mathrm{d}}[20] ;{ }^{\mathrm{e}}[19]$.

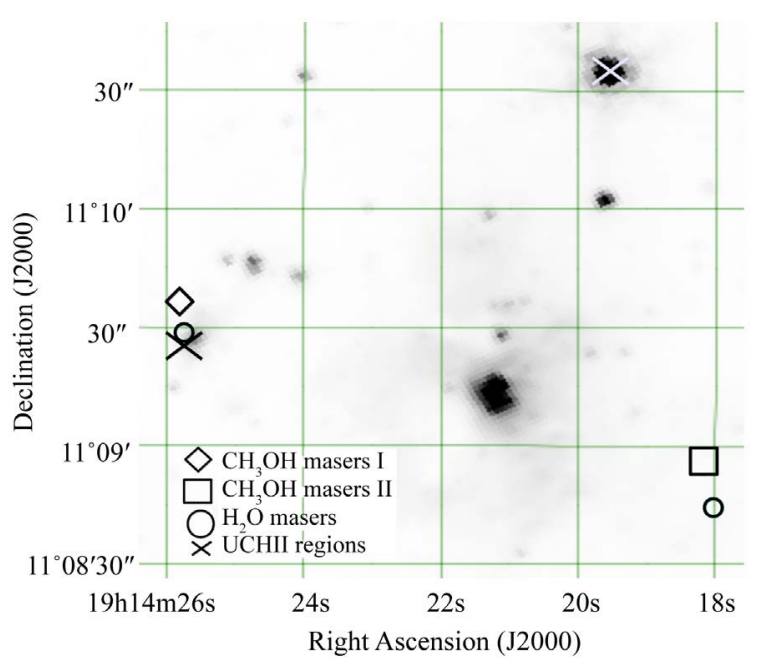

Figure 15. Figure showing the Class II and the Class I methanol maser and other sources of interest in region G45.47 + 0.07. The grayscale shows the $4.5 \mu \mathrm{m}$ Spitzer infrared image and the intensity range is between $0.385 \mathrm{MJy} \cdot \mathrm{sr}^{-1}$ and $538 \mathrm{MJy} \cdot \mathrm{sr}^{-1}$. The symbols are the same as in Figure 3.



Figure 16. Plot of intensities and center velocities $\left(v_{\mathrm{LSR}}\right)$ of methanol and $\mathrm{H}_{2} \mathrm{O}$ masers associated with $\mathrm{G} 45.47+0.07$. The symbols are the same as in Figure 4. 


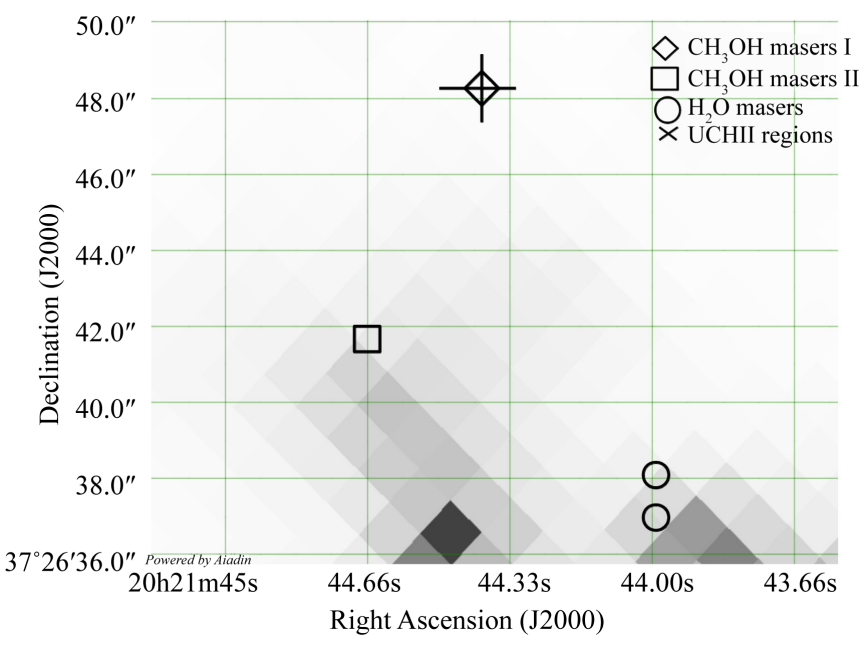

Figure 17. Figure showing the Class II and the Class I methanol maser and other sources of interest in region G75.78 + 0.34. The grayscale shows the $4.5 \mu \mathrm{m}$ Spitzer infrared image and the intensity range is between $0.536 \mathrm{MJy} \cdot \mathrm{sr}^{-1}$ and $797 \mathrm{MJy} \cdot \mathrm{sr}^{-1}$. The symbols are the same as in Figure 3.

G75.78 + 0.34 ([14]), this is equivalent to about 0.17 pc. Two $\mathrm{H}_{2} \mathrm{O}$ masers lie 10.0 " to the southwest of the Class I methanol maser. The positions of these sources, together with the telescopes used to observe them and the angular resolution (in terms of synthesized beam) of the observations, are listed in Table 8.

The center velocities $\left(v_{\mathrm{LSR}}\right)$ and intensities of the Class I and Class II methanol masers and $\mathrm{H}_{2} \mathrm{O}$ masers have been plotted in Figure 18. Two of the three $\mathrm{H}_{2} \mathrm{O}$ masers are at smaller $v_{\mathrm{LSR}}$ (i.e., blueshifted) than the Class II methanol maser. The Class I methanol maser is also blueshifted with respect to the Class II methanol maser.

\subsection{Regions for Which High Resolution Data Were Only Available for Class I and Class II Methanol Masers}

In Figure 2, we saw that for $60 \%$ of the regions we examined, high resolution data were present for the Class I and Class II methanol masers only, but not for the other associated tracers of star formation discussed in §3.2. The data on these methanol masers for such regions are presented in Table 9, including coordinates of each region, the offset in pc between the Class I and Class II methanol masers, and the characteristics of the spectral lines of each maser.

The projected distance between Class I and Class II methanol masers in these twenty-two regions is in general agreement with the separation between masers discussed in $\$ 3.3$ to $\S 3.10$. The maximum angular separation is 59.34" for G24.943 + 0.074; at a distance of $7.7 \mathrm{kpc}$ ([14]), this works out to $2.2 \mathrm{pc}$, similar to the projected distance between Class I and Class II masers in G45.47 + 0.07. The minimum projected separation was zero in three cases: G28.28 - 0.36, G37.47 - 0.11, G49.27 - 0.34, but that is likely due to the resolution of the observations not being great enough to resolve the Class I and Class II methanol maser sources. The mean projected separation between Class I and Class II methanol masers for all thirty regions discussed in this paper was 0.67 pc with a root mean squared error of $0.12 \mathrm{pc}$.

\section{Discussion}

In this section, we discuss the morphology and kinematics of the eight regions for which high resolution data were available. In $\S 4.1$, we identify three patterns of morphology and a notable trend in the center velocities of the masers. In §5 we describe three physical models which can explain the patterns discussed in §4.1.

\subsection{Morphology and Kinematics}

Of the thirty regions that we found to have data on Class I and Class II methanol masers, we were able to obtain 
Table 8. Positions and associated information for sources associated with G75.78 + 0.34

\begin{tabular}{|c|c|c|c|c|c|c|}
\hline Source type & R.A. (J2000) & Dec. (J2000) & Observing & Synthesized & Kinematic & \\
\hline & (h m s) & $\left({ }^{\circ}+"\right)$ & Telescope & Beam & $v_{\mathrm{LSR}}\left(\mathrm{km} \cdot \mathrm{s}^{-1}\right)$ & Intensity (Jy) \\
\hline $44 \mathrm{GHz}$ Class $\mathrm{I} \mathrm{CH}_{3} \mathrm{OH}$ maser ${ }^{\mathrm{a}}$ & 202144.4 & +372648 & VLA & $1.5^{\prime \prime} \times 1.5^{\prime \prime}$ & 3.8 & 8.7 \\
\hline $6.7 \mathrm{GHz}$ Class II $\mathrm{CH}_{3} \mathrm{OH}$ maser ${ }^{\mathrm{b}}$ & 202144.7 & +372642 & VLA & $0.5^{\prime \prime} \times 0.5^{\prime \prime}$ & -2.9 & 39 \\
\hline $22 \mathrm{GHz} \mathrm{H}_{2} \mathrm{O}$ maser $^{\mathrm{c}}$ & 202144.0 & +372638 & VLA & $0.52^{\prime \prime} \times 0.3^{\prime \prime}$ & 2.6 & 58 \\
\hline $22 \mathrm{GHz} \mathrm{H}_{2} \mathrm{O}$ maser & 202144.0 & +372637 & VLA & $0.52^{\prime \prime} \times 0.3^{\prime \prime}$ & 14 & 4.2 \\
\hline $22 \mathrm{GHz} \mathrm{H}_{2} \mathrm{O}$ maser & 202144.0 & +372637 & VLA & $0.52^{\prime \prime} \times 0.3^{\prime \prime}$ & 2.3 & 40 \\
\hline
\end{tabular}

${ }^{\mathrm{a}}[13] ;{ }^{\mathrm{b}}[26] ;{ }^{\mathrm{c}}[18]$.

Table 9. Regions with high resolution data for methanol masers only.

\begin{tabular}{|c|c|c|c|c|c|c|c|}
\hline \multirow[t]{2}{*}{ Region } & \multirow{2}{*}{$\begin{array}{l}\text { R.A. (J2000) } \\
\qquad(\mathrm{h} \mathrm{m} \mathrm{s})\end{array}$} & \multirow{2}{*}{$\begin{array}{c}\text { Dec. (J2000) } \\
\left({ }^{\circ} \prime \prime\right)\end{array}$} & \multirow{2}{*}{$\begin{array}{c}\text { MMI MMII offset } \\
\text { Telescope }\end{array}$} & \multicolumn{2}{|c|}{$44 \mathrm{GHz}$ Class I $\mathrm{CH}_{3} \mathrm{OH}$} & \multicolumn{2}{|c|}{ 6.7 GHz Class II $\mathrm{CH}_{3} \mathrm{OH}^{-}$} \\
\hline & & & & Beam & $v_{\mathrm{LSR}}\left(\mathrm{km} \cdot \mathrm{s}^{-1}\right)$ & Intensity (Jy) & (h m s) \\
\hline $\mathrm{G} 13.66-0.60^{\mathrm{a}}$ & 181722.9 & -172213.8 & $0.84 \pm 0.02$ & 48 & 28 & 51 & 33 \\
\hline $\mathrm{G} 19.36-0.02^{\mathrm{b}}$ & 182625.8 & -120356.9 & $0.96 \pm 0.06$ & 27 & 54 & 26 & 6.7 \\
\hline $\mathrm{G} 43.04-0.45^{\mathrm{a}}$ & 191138.8 & 084637.9 & $0.67 \pm 0.01$ & 58 & 3.8 & 55 & 10 \\
\hline G94.26- $0.41^{\mathrm{a}}$ & 213230.7 & 510214.9 & $0.31 \pm 0.01$ & -48 & 1.1 & -48 & 1.1 \\
\hline $\mathrm{G} 10.29-0.13^{\mathrm{b}}$ & 180849.5 & -200553.5 & $0.35 \pm 0.01$ & 14 & 2.0 & 10 & 2.7 \\
\hline $\mathrm{G} 14.89-0.40^{\mathrm{a}}$ & 18197.60 & -161125.6 & $0.61 \pm 0.01$ & 62 & 7.8 & 63 & 3.2 \\
\hline $\mathrm{G} 18.66+0.04^{\mathrm{b}}$ & 182453.7 & -123920.0 & $1.6 \pm 0.02$ & 80 & 2.1 & 80 & 22 \\
\hline $\mathrm{G} 18.89-0.47^{\mathrm{b}}$ & 18277.90 & -124135.5 & $0.01 \pm 0.01$ & 66 & 20 & 38 & 3.7 \\
\hline $\mathrm{G} 22.04+0.22^{\mathrm{b}}$ & 183034.7 & -093447.0 & $0.97 \pm 0.02$ & 51 & 32 & 54 & 6.1 \\
\hline $\mathrm{G} 23.96-0.11^{\mathrm{a}}$ & 183522.3 & -080128.0 & $0.72 \pm 0.02$ & 73 & 6.2 & 71 & 17 \\
\hline $\mathrm{G} 24.94+0.07^{\mathrm{b}}$ & 183631.5 & -070416.0 & $2.2 \pm 0.02$ & 41 & 1.7 & 53 & 4.2 \\
\hline $\mathrm{G} 25.27-0.43^{\mathrm{b}}$ & 183856.9 & -070048.0 & $0.03 \pm 0.01$ & 60 & 6.2 & 59 & 0.55 \\
\hline$G 28.28-0.36^{b}$ & 184413.2 & -04184.00 & $0.00 \pm 0.02$ & 48 & 4.1 & 81 & 62 \\
\hline $\mathrm{G} 28.84-0.25^{\mathrm{b}}$ & 184451.0 & -034549.0 & $1.1 \pm 0.02$ & 87 & 5.6 & 100 & 1.9 \\
\hline $\mathrm{G} 34.82+0.35^{\mathrm{a}}$ & 185337.7 & 015025.4 & $0.31 \pm 0.01$ & 57 & 0.67 & 56 & 0.27 \\
\hline $\mathrm{G} 36.11+0.55^{\mathrm{a}}$ & 185516.8 & 03056.70 & $1.0 \pm 0.01$ & 76 & 0.57 & 73 & 19 \\
\hline $\mathrm{G} 37.27+0.08^{\mathrm{a}}$ & 18593.73 & 035342.9 & $0.88 \pm 0.01$ & 90 & 1.0 & 90 & $\ldots^{d}$ \\
\hline G37.47 $-0.11^{b}$ & 19007.00 & 035953.0 & $0.00 \pm 0.01$ & 59 & 0.31 & 58 & 13 \\
\hline $\mathrm{G} 39.10+0.48^{\mathrm{b}}$ & 190058.1 & 054244.0 & $0.62 \pm 0.01$ & 27 & 11 & 15 & 15 \\
\hline G49.27 - $0.34^{b}$ & 19236.70 & 142013.0 & $0.00 \pm 0.01$ & 67 & 0.65 & 67 & $\ldots^{d}$ \\
\hline
\end{tabular}

${ }^{\mathrm{a}}[13] ;{ }^{\mathrm{b}}[17] ;{ }^{\mathrm{c}}[14] ;{ }^{\mathrm{d}}[14]$ reports a position fix only; ${ }^{\mathrm{e}}$ The values in this column were computed using a measurement of the distance from our sun to the region in question, the error indicates the propagated uncertainty in the determination of the heliocentric distance. Note: For a valid comparison across sources, the data has been rounded to the least number of significant figures.

high resolution data for associated star formation tracers for eight regions. Such associated data include $\mathrm{H}_{2} \mathrm{O}$ masers, ultracompact (UC) ionized hydrogen (H II) regions, and $4.5 \mu \mathrm{m}$ infrared data from Spitzer. For these eight regions, we looked at the morphology of Class I and II methanol masers, and $\mathrm{H}_{2} \mathrm{O}$ masers, and the center 


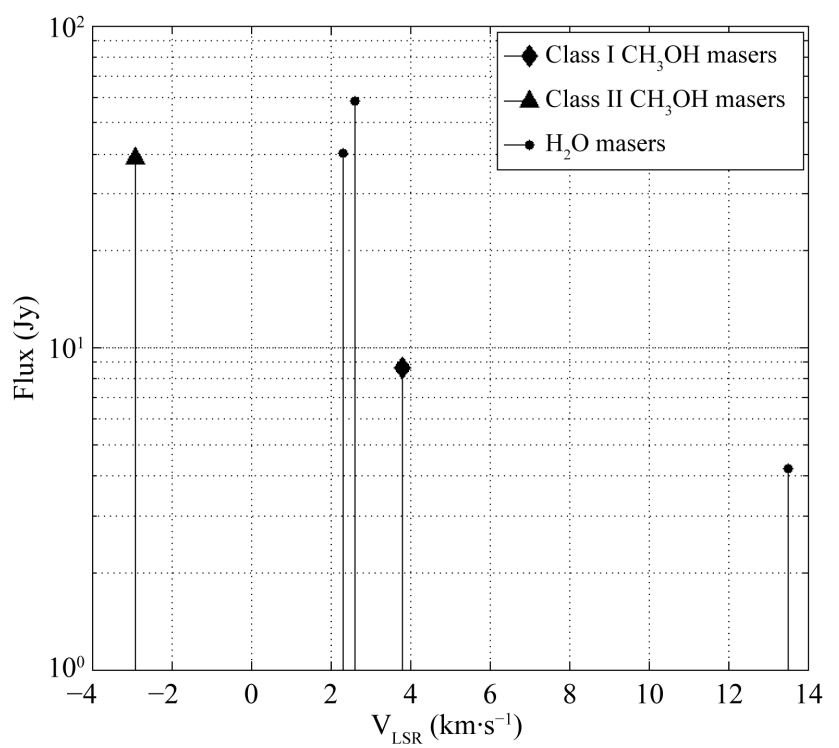

Figure 18. Plot of intensities and center velocities $\left(v_{\mathrm{LSR}}\right)$ of methanol and $\mathrm{H}_{2} \mathrm{O}$ masers associated with $\mathrm{G} 75.78+0.34$. The symbols are the same as in Figure 4.

velocities of the line profiles of these masers. We also looked at the location of the UC H II regions and $4.5 \mu \mathrm{m}$ infrared sources. Based on our examination of these data, we have identified three distinct patterns in the morphology of the masers, and one notable trend in the center velocities of the Class I methanol and $\mathrm{H}_{2} \mathrm{O}$ maser spectral lines in relation to the center velocity of the Class II methanol masers.

\subsection{Linear Arrangement of $\mathrm{H}_{2} \mathrm{O}$ Masers}

In two of the eight sources, G9.62 + 0.19 and $\mathrm{G} 45.07+0.13$, the $\mathrm{H}_{2} \mathrm{O}$ masers are in a linear arrangement, spread out along a straight line (approximately) between the Class I and Class II methanol maser (Figure 3 and Figure 13). There are nine $\mathrm{H}_{2} \mathrm{O}$ masers in G9.62 + 0.19, but only five in $\mathrm{G} 45.07+0.13$, so the linear structure is more pronounced in G9.62 + 0.19. The projected separation between the two classes of methanol maser is $0.27 \mathrm{pc}$ for G9.62 + 0.19 and 0.17 pc for G45.07 + 0.13. A UC H II region is almost coincident with the Class II methanol maser in both sources. Since UC H II regions are formed by hot and massive young protostars, it is reasonable to conclude that the same ionizing radiation that is producing the UC H II region is also likely providing the radiative excitation of the Class II methanol maser. The linear arrangement of $\mathrm{H}_{2} \mathrm{O}$ masers between the Class II and Class I methanol masers in both G9.62 + 0.19 and G45.07 + 0.13 is also suggestive of an outflow, with the Class I methanol maser excited near the head of the outflow in both sources. As stated in §3.3, we consider masers with $v_{\text {LSR }}$ larger than the $v_{\text {LSR }}$ of the Class II methanol maser to be redshifted. Seven of the nine $\mathrm{H}_{2} \mathrm{O}$ masers in G9.62 + 0.19 are redshifted with respect to the Class II methanol maser (e.g., see Table 1, where the $v_{\text {LSR }}$ of $7 \mathrm{H}_{2} \mathrm{O}$ masers are larger than the $v_{\text {LSR }}$ of the Class II methanol maser, and Figure 4). Therefore, these 7 masers are likely in the redshifted lobe of the outflow (see $\$ 5.1$ for additional discussion). The Class I methanol maser also has a larger $v_{\mathrm{LSR}}$ than the $v_{\mathrm{LSR}}$ of the Class II maser, so it can also be located in the redshifted lobe of the outflow. Likewise, in G45.07 + 0.13, three out of four masers are redshifted with respect to the Class II methanol maser, as is the Class I methanol maser (Table 6 and Figure 14).

In G9.62 + 0.19, however, we cannot rule out that the Class I methanol maser may not be associated with the Class II methanol maser, particularly given the recent discovery by [27] that suggests that Class I masers may also be excited in shocks driven by expanding H II regions. Indeed, there is a UC H II region to the southwest of the Class I methanol maser in G9.62 + 0.19 and a $4.5 \mu \mathrm{m}$ infrared source near the UC H II region, indicating another center of high mass star formation activity at this location (Figure 3). Still, with 7 of $9 \mathrm{H}_{2} \mathrm{O}$ masers in G9.62 +0.19 redshifted with respect to the Class II maser, the outflow interpretation is reasonable, so that the data are certainly compatible with disk-outflow systems. 


\section{3. $\mathrm{H}_{2} \mathrm{O}$ Masers Clustered Near Class II Methanol Maser}

In two of the eight regions, G10.47 + 0.03 and G75.78 + 0.34, the $\mathrm{H}_{2} \mathrm{O}$ masers are far away from the Class I methanol maser (Figure 5 and Figure 17). In G10.47 + 0.03, all the three $\mathrm{H}_{2} \mathrm{O}$ masers are near the Class II methanol maser. A faint $4.5 \mu \mathrm{m}$ source and a UC H II region are coincident with the Class II and $\mathrm{H}_{2} \mathrm{O}$ maser position. As stated in $\S 3.3$, we consider masers with $v_{\mathrm{LSR}}$ smaller than the $v_{\mathrm{LSR}}$ of the Class II methanol maser to be blueshifted. All three $\mathrm{H}_{2} \mathrm{O}$ masers in $\mathrm{G} 10.47+0.03$, and the Class I methanol maser, are blueshifted with respect to the Class II methanol maser (Table 2 and Figure 6).

In $\mathrm{G} 75.78+0.34$, all three $\mathrm{H}_{2} \mathrm{O}$ masers are at $~ 9.1$ pc from the Class II methanol maser, but a line from the Class II methanol maser to the $\mathrm{H}_{2} \mathrm{O}$ masers is roughly perpendicular to a line joining the Class I and II methanol masers (Figure 17). An extended $4.5 \mu \mathrm{m}$ source straddles the Class II methanol maser and $\mathrm{H}_{2} \mathrm{O}$ maser positions. All three $\mathrm{H}_{2} \mathrm{O}$ masers, and the Class I methanol maser, are redshifted with respect to the Class II methanol maser (Table 8 and Figure 18). We speculate that the Class II methanol maser and $\mathrm{H}_{2} \mathrm{O}$ masers are located in a circumstellar disk in both these sources, and discuss this further in $§ 5.2$.

\section{4. $\mathrm{H}_{2} \mathrm{O}$ Masers Clustered Near Class I Methanol Maser}

In three of the eight sources, G12.20 - 0.11, G31.41 + 0.31, and G35.03 + 0.35, the $\mathrm{H}_{2} \mathrm{O}$ masers are far away from the Class II methanol maser, but are located near the Class I methanol maser (Figure 7, Figure 9, and Figure 11). Of the 6 masers near the Class I methanol maser in $\mathrm{G} 12.20-0.11$, two $\mathrm{H}_{2} \mathrm{O}$ masers, and the Class I methanol maser, are redshifted with respect to the Class II methanol maser (Table 3 and Figure 8). Meanwhile, three $\mathrm{H}_{2} \mathrm{O}$ masers are blueshifted, and one has the same center velocity, as the Class II methanol maser. Of the 8 masers in G31.41 + 0.31, five $\mathrm{H}_{2} \mathrm{O}$ masers, and the Class I methanol maser, are blueshifted with respect to the Class II methanol maser, whereas two $\mathrm{H}_{2} \mathrm{O}$ masers are redshifted, and one has the same $v_{\mathrm{LSR}}$ as the Class II methanol maser (Table 4 and Figure 10). There is only one $\mathrm{H}_{2} \mathrm{O}$ maser in $\mathrm{G} 35.03+0.35$, and it, along with the Class I methanol maser, is redshifted with respect to the Class II water maser (Table 5 and Figure 12). In all the three regions, G12.20 - 0.11, G31.41 + 0.31, and G35.03 + 0.35, there is a $4.5 \mu \mathrm{m}$ source that is closer to the Class I methanol maser than the Class II methanol maser. In §5.3, we will ascribe the geometry in these sources to one or more outflows.

\subsection{The Eighth Source G45.47 + 0.07}

The eighth source for which we have high angular resolution data for $\mathrm{H}_{2} \mathrm{O}$ masers and other associated tracers, G45.47 + 0.07, does not fit into any of the three categories listed above. It has one $\mathrm{H}_{2} \mathrm{O}$ maser near the Class I methanol maser source, and one $\mathrm{H}_{2} \mathrm{O}$ maser near the Class II methanol maser source (Figure 15). The Class I methanol maser, an $\mathrm{H}_{2} \mathrm{O}$ maser near it, and the $\mathrm{H}_{2} \mathrm{O}$ maser near the Class II methanol maser, are all redshifted with respect to the Class II methanol maser (Table 7 and Figure 16). We interpret this as a hybrid of the models discussed in $\S 4.3$ and $\S 4.4$, and discuss it further in $\S 5.4$.

\section{Models Based on High Angular Resolution Data}

Based on our findings (§3) and discussion in §4.1, we have constructed three disk-outflow models for methanol masers in star forming regions. As discussed in $\S 1$, star formation is accompanied by a circumstellar disk from which the central protostar accretes material, and a bipolar outflow at right angles to the circumstellar disk which carries mass and angular momentum away from the protostar (Figure 19). In general, the disk may be inclined at some angle to the line of sight, in which case one lobe of the outflow would be redshifted and the other blueshifted. We will now discuss each model in detail.

\subsection{Disk-Outflow Model 1}

Our first model is based on the morphology of methanol and $\mathrm{H}_{2} \mathrm{O}$ masers in $\mathrm{G} 9.62+0.19$ and $\mathrm{G} 45.07+0.13$ (§3.3, §3.8, and §4.2). Figure 20 shows the model of the disk and outflow, together with the locations of the Class II and Class I methanol masers, and the $\mathrm{H}_{2} \mathrm{O}$ masers. We have chosen to put the information from the G9.62 + 0.19 star forming region (§3.3) in the figure. Since it is known that Class II methanol masers are usually found near protostars, we have put the disk near the Class II methanol maser source. Next, it is well known that 


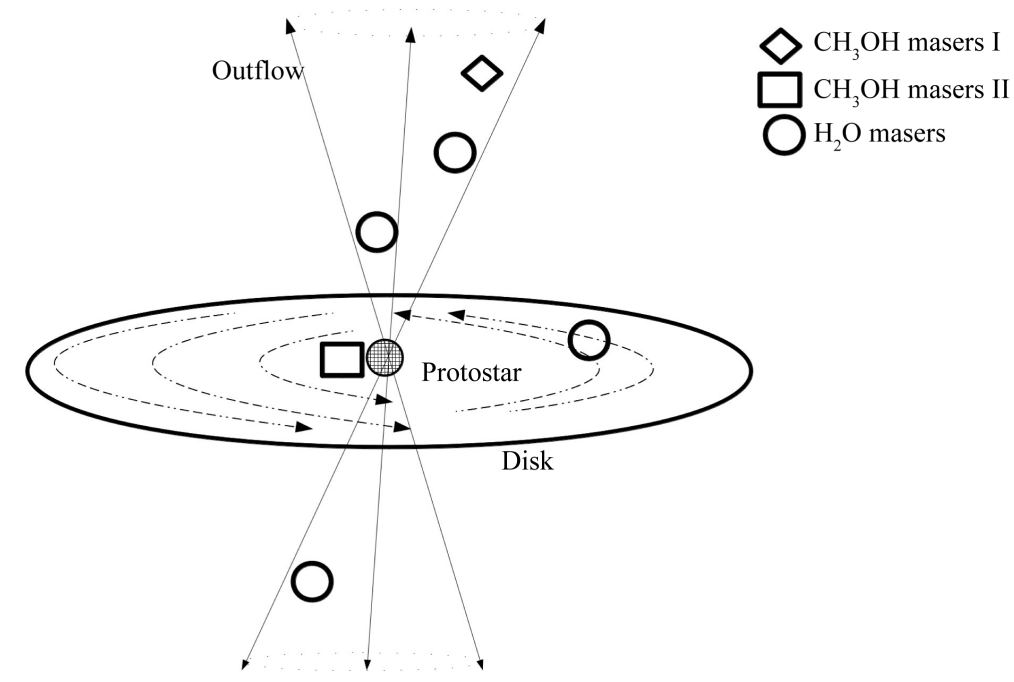

Figure 19. Diagram showing an accretion disk and outflow from a massive young protostar. As discussed in $\S 1$, star formation is accompanied by a circumstellar disk from which the central protostar accretes material, and a bipolar outflow at right angles to the disk which carries mass and angular momentum away from the protostar. Using the same symbols as were used in §3 we have shown some typical locations for Class I and Class II methanol masers and water masers in relation to the circumstellar disk, outflow and massive protostar. In general, the disk may be inclined at some angle to the line of sight, in which case one lobe of the outflow would be redshifted and the other blueshifted.

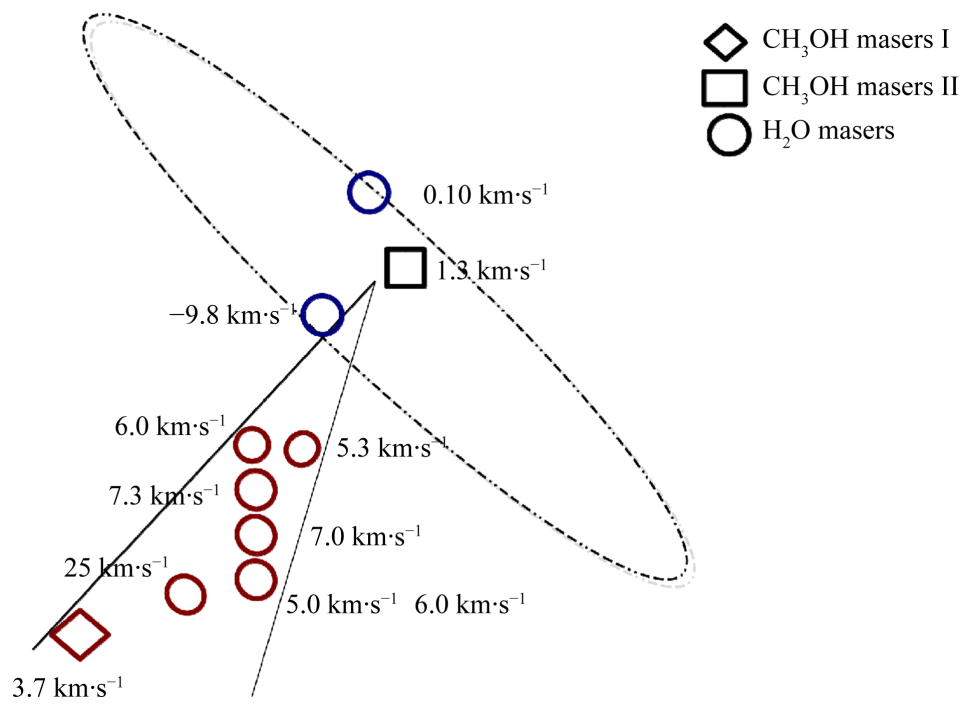

Figure 20. Diagram of our disk-outflow model 1, showing the locations of the Class II and I methanol masers and the $\mathrm{H}_{2} \mathrm{O}$ masers. We have chosen to put the information from the G9.62 + 0.19 star forming region (Figure 3 and Table 1) in this figure. The symbols are the same as those used in §3, and are marked at the top right of the figure. Masers that are redshifted with respect to the Class II methanol maser are shown in red.

$\mathrm{H}_{2} \mathrm{O}$ masers are formed in both outflows and circumstellar disks in high mass star forming regions. Except for the two water masers nearest to the Class II methanol maser in G9.62 + 0.19, all the other 7 masers are redshifted (i.e., at higher center velocities, $v_{\mathrm{LSR}}$ ) with respect to the Class II methanol maser, and well separated 
from it in position, as is the Class I methanol maser. Therefore, we interpret all of them to be part of the redshifted lobe of the outflow. We have used the position of the $\mathrm{H}_{2} \mathrm{O}$ maser on the extreme right and the Class I methanol maser on the left in G9.62 + 0.19 to constrain the edges of the redshifted outflow lobe, giving us a nominal opening angle of $23^{\circ}$ for the outflow. Choosing the edges of the two redshifted $\mathrm{H}_{2} \mathrm{O}$ masers in G45.07 + 0.13 also gives us a similar opening angle for the outflow. In both regions, therefore, the outflow appears to be well collimated. This is usually the case in low mass star forming regions in the earlier stages of star formation, and has been known to be true in high mass star forming regions whenever outflows can be disentangled between sources ([3]). In other words, due to the multiplicity of sources in high mass star forming regions, it is usually difficult to tell which outflow is coming from which source. Here, however, if all the redshifted masers are truly part of the same redshifted outflow lobe that is centered near the Class II methanol maser location, then the smaller value of the collimation angle that we have obtained is as expected. For example, in low mass star forming regions, [28] described collimation angles of a few degrees, and in high mass star forming regions, [29] found outflows collimated to the same degree as in low mass star forming regions. The two blueshifted $\mathrm{H}_{2} \mathrm{O}$ masers in Figure 20 near the Class II methanol maser in G9.62 + 0.19 may be part of the blueshifted lobe of the outflow or may be part of the circumstellar disk of the protostar. We favor the latter interpretation for the southeastern blue shifted maser, at least, since the blueshifted lobe should be opposite the redshifted lobe. Likewise the redshifted and blueshifted masers that are almost coincident with the Class II methanol maser in G45.07 + 0.13 (Figure 13 and Table 6) could be interpreted as being at the base of the redshifted and blueshifted outflow lobes, or they could be in the circumstellar disk around the protostar.

While the model of the disk and the redshifted lobe given above is one interpretation of the data, it is worth noting that the morphology of methanol and $\mathrm{H}_{2} \mathrm{O}$ masers in $\mathrm{G} 9.62+0.19$ in particular, does support other geometries that we have not considered here. For example, [27] have suggested that some Class I methanol masers may be excited in shocks driven by expanding $\mathrm{H}$ II regions. We note that there is a UC H II region to the southwest of the Class I methanol maser in G9.62 + 0.19 (Figure 3) which could be responsible for the shock causing the Class I methanol maser.

\subsection{Disk-Outflow Model 2}

The second model is based on the morphology of methanol and $\mathrm{H}_{2} \mathrm{O}$ masers in $\mathrm{G} 10.47+0.03$ and G75.78 + 0.34 (§3.4, §3.10, and §4.3). Figure 21 shows the model of the disk and outflow, together with the locations of the Class II and Class I methanol masers, and the $\mathrm{H}_{2} \mathrm{O}$ masers. We have chosen to put the information from $\mathrm{G} 10.47+0.03$ (§3.4) into Figure 21. In both these sources, we interpret the $\mathrm{H}_{2} \mathrm{O}$ masers to be located in a circumstellar disk together with the Class II methanol maser, with the Class I methanol maser located in the outflow. This is a reasonable interpretation for, where the $\mathrm{H}_{2} \mathrm{O}$ masers are clustered close to the Class II methanol maser (Figure 5). In G75.78 + 0.34, however, while the geometry suggests such an interpretation (Figure 17), the size of the circumstellar disk would be too large, if it were to be constrained by the distance between the $\mathrm{H}_{2} \mathrm{O}$ masers and the Class II methanol maser, which is $0.2 \mathrm{pc} \equiv 40,000 \mathrm{AU}$ (e.g., compare to the circumstellar disk observed by [30] in Cepheus A with a 300 AU radius). Therefore, it is more likely that either the Class I and II methanol masers are associated in G75.78 + 0.34, and the $\mathrm{H}_{2} \mathrm{O}$ masers are part of an outflow or disk of another system (e.g., there is a $4.5 \mu \mathrm{m}$ peak near the $\mathrm{H}_{2} \mathrm{O}$ masers), or that the Class II methanol and $\mathrm{H}_{2} \mathrm{O}$ masers are associated, and the Class I methanol maser is part of an outflow from a nearby source. Recently [31] observed this region with the ATCA working at $22 \mathrm{Ghz}$ and found strong evidence of a high velocity ( $v_{\text {LSR }}$ approaching $100 \mathrm{~km} \cdot \mathrm{s}^{-1}$ ) outflow. [31] was published after the thesis ([2]) on which this paper is based was defended, while this paper was in preparation, and therefore their data was not used.

\subsection{Disk-Outflow Model 3}

Our third model is based on the morphology of methanol and $\mathrm{H}_{2} \mathrm{O}$ masers in G12.20 - 0.11, G31.41 + 0.31, and G35.03 + 0.35 (§3.5, §3.6, §3.7, and §4.4). Figure 22 shows the model of the disk and outflow, together with the locations of the Class II and Class I methanol masers and the $\mathrm{H}_{2} \mathrm{O}$ masers. We have chosen to put the information from G31.41 + 0.31 (§3.6) in Figure 22. In G31.41 + 0.31, $5 \mathrm{H}_{2} \mathrm{O}$ masers are blueshifted, and 2 $\mathrm{H}_{2} \mathrm{O}$ masers are redshifted, with respect to the Class II methanol maser (Table 4). The Class I methanol maser is blueshifted with respect to the Class II methanol maser, and has a very similar $v_{\mathrm{LSR}}$ to the $v_{\mathrm{LSR}}$ of the $\mathrm{H}_{2} \mathrm{O}$ masers. Therefore, we have put the Class II methanol maser at the protostar and the Class I methanol maser along with 


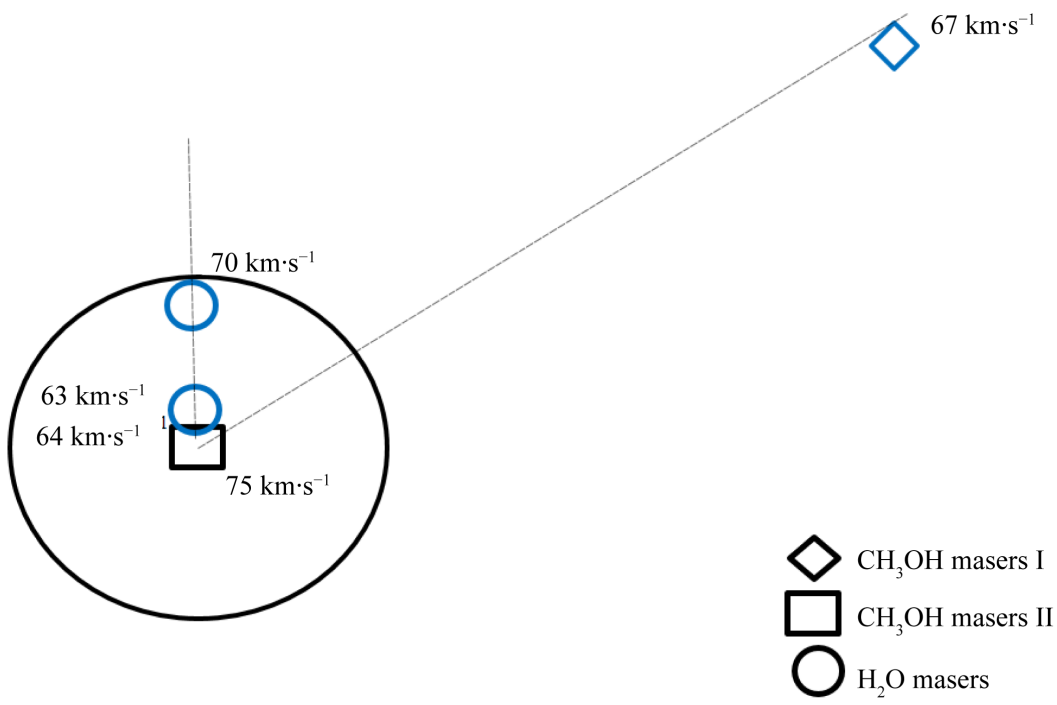

Figure 21. Diagram of our disk-outflow model 2, showing the locations of the Class II and I methanol masers and the $\mathrm{H}_{2} \mathrm{O}$ masers. We have chosen to put the information from the G10.47 + 0.03 star forming region (Figure 5 and Table 2) in this figure. The symbols are the same as those used in §3, and are marked at the bottom right of the figure. Masers that are blueshifted with respect to the Class II methanol maser are shown in blue.

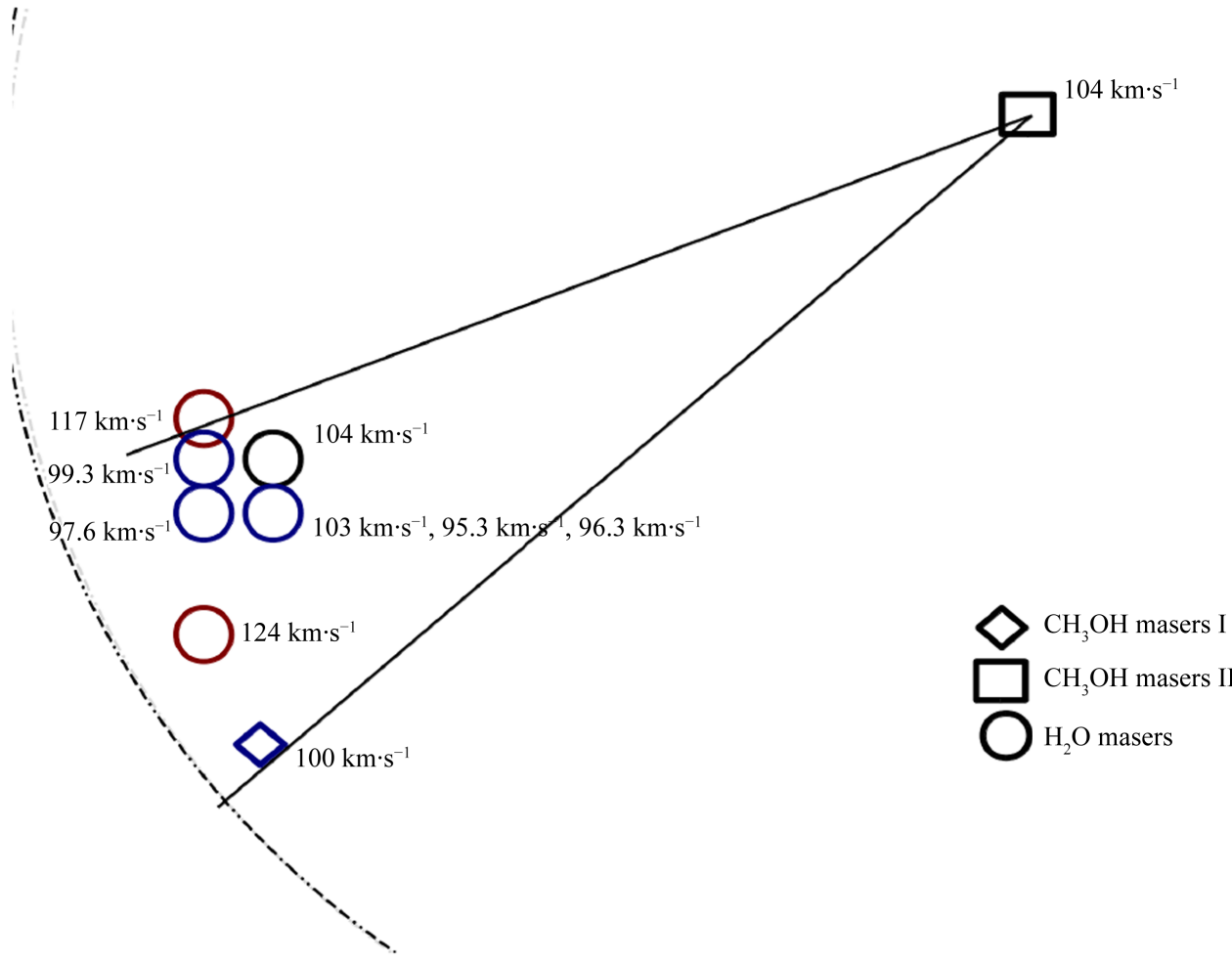

Figure 22. Diagram of our disk-outflow model 3, showing the locations of the Class II and I methanol masers and the $\mathrm{H}_{2} \mathrm{O}$ masers. We have chosen to put the information from the G31.41 + 0.31 star forming region (Figure 9 and Table 4) in this figure. The symbols are the same as those used in $\S 3$, and are marked at the bottom right of the figure. Masers that are redshifted with respect to the Class II methanol maser are shown in red. The dot-dashed line is meant to be visually suggestive of a bow shock driven into the ambient interstellar material. 
the blueshifted $\mathrm{H}_{2} \mathrm{O}$ masers in the blueshifted lobe of an outflow in Figure 22. Based on the position of the Class I methanol maser and the northern-most blueshifted $\mathrm{H}_{2} \mathrm{O}$ maser, we get an opening angle for the outflow equal to $22^{\circ}$, indicating that the outflow is well collimated. The situation is complicated, however, by the presence of the two redshifted water masers. Clearly, they cannot be in the same outflow as the blueshifted water masers. It is likely, therefore, that they are in the redshifted lobe of a different outflow. Indeed, there is a UC HII region to the northeast, and a $4.5 \mu \mathrm{m}$ source to the northwest of the redshifted $\mathrm{H}_{2} \mathrm{O}$ masers (Figure 9), and either could be the source of the redshifted lobe of an outflow.

A similar model works for G35.03 + 0.35, except that the Class I methanol and the $\mathrm{H}_{2} \mathrm{O}$ maser are redshifted with respect to the Class II methanol maser (Table 5), so we are seeing the redshifted lobe of an outflow in this region. Since there is only one $\mathrm{H}_{2} \mathrm{O}$ maser, we cannot constrain the opening angle of the outflow. The interpretation is more difficult in G12.20 - 0.11, where the Class I methanol maser and two $\mathrm{H}_{2} \mathrm{O}$ masers are redshifted with respect to the Class II methanol maser, but there are now four blueshifted $\mathrm{H}_{2} \mathrm{O}$ masers (Table 3). Again, it is clear that there must be two outflows in this region, and if we associate the redshifted $\mathrm{H}_{2} \mathrm{O}$ masers and Class I methanol maser with the redshifted lobe of one outflow centered on the protostar near the Class II methanol maser, the blueshifted $\mathrm{H}_{2} \mathrm{O}$ masers must be in the blueshifted lobe of another outflow. Indeed, there is a $4.5 \mu \mathrm{m}$ source to the east of the blueshifted water masers, and another one to the southeast (Figure 7), that could be the driving sources of the blueshifted outflow. The opening angles of the outflows are $11^{\circ}$ and $19^{\circ}$.

In summary, all three of these regions suggest a model in which the Class I methanol maser and the $\mathrm{H}_{2} \mathrm{O}$ masers are located at the head of outflows where the outflow runs into ambient interstellar material. However, other models where either the Class I and II methanol maser, or one type of methanol maser and $\mathrm{H}_{2} \mathrm{O}$ maser, are not associated with each other, cannot be ruled out.

\subsection{Hybrid Model for G45.47 + 0.07}

Our 8th source, G45.47+0.07 (§3.9 and §4.5) is likely a hybrid of the disk-outflow model 2 (§5.2) and diskoutflow model 3 (§5.3). The Class I methanol maser and the $\mathrm{H}_{2} \mathrm{O}$ maser near it are both redshifted with respect to the Class II methanol maser, so they can be located in the redshifted lobe of an outflow (disk-outflow model 3; §5.3). Meanwhile, the Class II methanol maser and the other $\mathrm{H}_{2} \mathrm{O}$ maser could be in the disk (disk-outflow model 2; §5.2).

\section{Conclusions}

The primary motivation for this paper was to find out whether disk-outflow systems are compatible with high mass star formation, based on the existing data on methanol masers, along with other associated tracers of star formation. We searched the literature for high angular resolution data on Class I and Class II methanol masers, $\mathrm{H}_{2} \mathrm{O}$ masers, UC H II regions, and $4.5 \mu \mathrm{m}$ infrared sources. We wanted the highest angular resolution data wherever available, so we looked for VLA (or, even better, VLBI) data. We started with 44 Class I methanol maser sources observed with the VLA and listed in [11]. By searching the literature for data around these locations, we obtained high angular resolution data on Class II methanol masers, $\mathrm{H}_{2} \mathrm{O}$ masers, UC H II regions, and $4.5 \mu \mathrm{m}$ infrared sources.

We found a total of thirty regions that contained both Class I and II methanol masers. For twenty two of these regions high angular resolution data were only available on the methanol masers. For these regions, we produced a table of the available data and the projected distances between the Class I and Class II methanol masers (Table 9). For the remaining eight regions, high resolution data were available on Class I and II methanol masers, water masers, UC H II regions and $4.5 \mu \mathrm{m}$ infrared sources. For these eight regions, we prepared maps of the positions of the Class I and II methanol masers, and the other star formation tracers listed above ( $\$ 3.3$ §3.10). We also prepared plots reflecting the center velocities $\left(v_{\mathrm{LSR}}\right)$ of the Class I and Class II methanol and $\mathrm{H}_{2} \mathrm{O}$ masers.

Based on these plots, we proposed three disk-outflow models. In all three models, we placed the Class II methanol maser at the location of the protostar, and the Class I methanol maser in the outflow. In our first diskoutflow model (§5.1), the $\mathrm{H}_{2} \mathrm{O}$ masers are in a linear pattern delineating the outflow. Two of the eight regions are consistent with this model, although alternative scenarios cannot be ruled out. In our second disk-outflow model (§5.2), the $\mathrm{H}_{2} \mathrm{O}$ masers are located in a circumstellar disk near the Class II methanol maser location; two regions are consistent with this model. In our third disk-outflow model (§5.3), the $\mathrm{H}_{2} \mathrm{O}$ masers are located in one 
or more outflows at the edge of the shocked region where the outflow interacts with the ambient interstellar medium; three regions are consistent with this model. Finally, the 8th region is a hybrid of two of these models with one $\mathrm{H}_{2} \mathrm{O}$ maser located near the Class I methanol maser in the outflow and another in the circumstellar disk near the Class II methanol maser (\$5.4). All eight regions are therefore compatible with disk-outflow models, with the Class I methanol maser in the outflow, and the Class II methanol maser near the protostar.

Together, these models show the utility of coordinated high angular resolution observations of methanol masers. Since new receivers at $36 \mathrm{GHz}$ are now available at the VLA, there is excellent scope for unique insight into high mass star formation through future observations of Class I and Class II methanol masers.

\section{References}

[1] Breen, S.L., Ellingsen, S.P., Contreras, Y., et al. (2013) Confirmation of the Exclusive Association between 6.7-GHz Methanol Masers and High-Mass Star Formation Regions. Monthly Notices of the Royal Astronomical Society, 435, 524-530. http://dx.doi.org/10.1093/mnras/stt1315

[2] Farmer, H.F. (2013) Insights into High Mass Star Formation from Methanol Maser Observations. Master’s Thesis, DePaul University, Chicago. http://via.library.depaul.edu/csh_etd/45

[3] McKee, C.F. and Ostriker, E.C. (2007) Theory of Star Formation. Annual Review of Astronomy and Astrophysics, 45, 565-687. http://dx.doi.org/10.1146/annurev.astro.45.051806.110602

[4] Loinard, L., Torres, R.M., Mioduszewski, A.J. and Rodrı'guez, L.F. (2008) A Preliminary VLBA Distance to the Core of Ophiuchus, with an Accuracy of 4\%. The Astrophysical Journal, 675, L29-L32.

[5] Genzel, R., Reid, M.J., Moran, J.M. and Downes, D. (1981) Proper Motions and Distances of H2O Maser Sources. I-The Outflow in Orion-KL. Astrophysical Journal, 244, 884-902. http://dx.doi.org/10.1086/158764

[6] Zinnecker, H. and Yorke, H.W. (2007) Toward Understanding Massive Star Formation. Annual Review of Astronomy and Astrophysics, 45, 481-563. http://dx.doi.org/10.1146/annurev.astro.44.051905.092549

[7] Menten, K. (1991) Methanol Masers and Submillimeter Wavelength Water Masers in Star-Forming Regions. In: Haschick, A.D. and Ho, P.T.P., Eds., Atoms, Ions and Molecules: New Results in Spectral Line Astrophysics, Astronomical Society of the Pacific, San Francisco, 119.

[8] Sobolev, A.M., Ostrovskii, A.B., Kirsanova, M.S., Shelemei, O.V., Voronkov, M.A. and Malyshev, A.V. (2006) Methanol Masers and Star Formation. arXiv:astro-ph/0601260.

[9] Cragg, D.M., Johns, K.P., Godfrey, P.D. and Brown, R.D. (1992) Pumping the Interstellar Methanol Masers. Monthly Notices of the Royal Astronomical Society, 259, 203-208.

[10] Voronkov, M. (2013) Australia Telescope National Facility. http://www.atnf.csiro.au/news/newsletter/feb05/New_class-1_methanol_masers.htm

[11] Val’tts, I.E. and Larionov, G.M. (2007) A General Catalog of Class I Methanol Masers. Astronomy Reports, 51, 519530. http://dx.doi.org/10.1134/S1063772907070013

[12] Cyganowski, C.J., Brogan, C.L., Hunter, T.R. and Churchwell, E. (2011) Deep Very Large Array Radio Continuum Surveys of GLIMPSE Extended Green Objects (EGOs). The Astrophysical Journal, 743, 56. http://dx.doi.org/10.1088/0004-637X/743/1/56

[13] Kurtz, S., Hofner, P. and Alvarez, C.V. (2004) A Catalog of $\mathrm{CH}_{3} \mathrm{OH} 7_{0}-6_{1} \mathrm{~A}^{+}$Maser Sources in Massive Star-Forming Regions. The Astrophysical Journal Supplement, 155, 149. http://dx.doi.org/10.1086/423956

[14] Pestalozzi, M.R., Minier, V. and Booth, R.S. (2005) A General Catalogue of 6.7-GHz Methanol Masers. I. Data. Astronomy \& Astrophysics, 432, 737-742. http://dx.doi.org/10.1051/0004-6361:20035855

[15] Caswell, J.L., Vaile, R.A., Ellingsen, S.P., Whiteoak, J.B. and Norris, R.P. (1995) Galactic Methanol Masers at 6.6 GHz. Monthly Notices of the Royal Astronomical Society, 272, 96-138.

[16] Pandian, J.D., Momjian, E., Xu, Y., Menten, K.M. and Goldsmith, P.F. (2011) The Arecibo Methanol Maser Galactic Plane Survey. IV. Accurate Astrometry and Source Morphologies. The Astrophysical Journal, 730, Article ID: 55. http://dx.doi.org/10.1088/0004-637X/730/1/55

[17] Cyganowski, C.J., Brogan, C.L., Hunter, T.R. and Churchwell, E. (2009) A Class I and Class II CH $\mathrm{CH}_{3} \mathrm{OH}$ Maser Survey of EGOs from the GLIMPSE Survey. The Astrophysical Journal, 702, 1615. http://dx.doi.org/10.1088/0004-637X/702/2/1615

[18] Hofner, P. and Churchwell, E. (1996) A Survey of Water Maser Emission toward Ultracompact HII Regions. Astronomy and Astrophysics Supplement Series, 120, 283.

[19] Wood, D.O.S. and Churchwell, E. (1989) The Morphologies and Physical Properties of Ultracompact H II Regions. Astronomy and Astrophysics Supplement Series, 69, 831-895. http://dx.doi.org/10.1086/191329 
[20] Urquhart, J.S., Hoare, M.G., Purcell, C.R., et al. (2009) The RMS Survey: 6 cm Continuum VLA Observations towards Candidate Massive YSOs in the Northern Hemisphere. Astronomy \& Astrophysics, 501, 539-551. http://dx.doi.org/10.1051/0004-6361/200912108

[21] Churchwell, E., Babler, B.L., Meade, M.R., et al. (2009) The Spitzer/GLIMPSE Surveys: A New View of the Milky Way. Publications of the Astronomical Society of the Pacific, 121, 213-230. http://dx.doi.org/10.1086/597811

[22] Cyganowski, C.J., Whitney, B.A., Holden, E., et al. (2008) A Catalog of Extended Green Objects in the GLIMPSE Survey: A New Sample of Massive Young Stellar Object Outflow Candidates. The Astrophysical Journal, 136, 2391. http://dx.doi.org/10.1088/0004-6256/136/6/2391

[23] Sanna, A., Reid, M.J., Moscadelli, L., Dame, T.M., Menten, K.M. and Brunthaler, A. (2009) Trigonometric Parallaxes of Massive Star-Forming Regions. VII. G9.62 + 0.20 and the Expanding 3 kpc ARM. The Astrophysical Journal, 706, 464. http://dx.doi.org/10.1088/0004-637X/706/1/464

[24] Forster, J.R. and Caswell, J.L. (2000) Radio Continuum Emission at $\mathrm{OH}$ and $\mathrm{H}_{2} \mathrm{O}$ Maser Sites. The Astrophysical Journal, 530, 371. http://dx.doi.org/10.1086/308347

[25] Minier, V., Conway, J.E. and Booth, R.S. (2001) VLBI Observations of 6.7 and $12.2 \mathrm{GHz}$ Methanol Masers toward High Mass Star-Forming Regions. II. Tracing Massive Protostars. Astronomy \& Astrophysics, 369, 278-290. http://dx.doi.org/10.1051/0004-6361:20010124

[26] Caswell, J.L., Vaile, R.A. and Forster, J.R. (1995) Methanol and Hydroxyl Maser Positions. Monthly Notices of the Royal Astronomical Society, 277, 210-216.

[27] Voronkov, M.A., Caswell, J.L., Ellingsen, S.P. and Sobolev, A.M. (2010) New 9.9-GHz Methanol Masers. Monthly Notices of the Royal Astronomical Society, 405, 2471-2484. http://dx.doi.org/10.1111/j.1365-2966.2010.16624.x

[28] Arce, H.G., Shepherd, D., Gueth, F., Lee, C.-F., Bachiller, R., Rosen, A. and Beuther, H. (2007) Molecular Outflows in Low- and High-Mass Star-forming Regions. In: Protostars \& Planets, University of Arizona Press, Tucson, 245-260.

[29] Beuther, H. (2005) X-Ray and Radio Connections. In: Sjouwerman, L.O. and Dyer, K. K., Electronically Published by NRAO. http://www.aoc.nrao.edu/events/xraydio/[meetingcont/1.1_kirk.pdf

[30] Torrelles, J.M., Gomez, J.F., Rodriguez, L.F., et al. (1996) The Thermal Radio Jet of Cepheus A HW2 and the Water Maser Distribution at 0"08 Scale (60 AU). Astrophysical Journal Letters, 457, L107. http://dx.doi.org/10.1086/309903

[31] Titmarsh, A.M., Ellingsen, S.P., Breen, S.L., Caswell, J.L. and Voronkov, M.A. (2013) G 10.472 + 0.027: An Extreme Water Maser Outflow Associated with a Massive Protostellar Cluster. Astrophysical Journal Letters, 775, L12. http://dx.doi.org/10.1088/2041-8205/775/1/L12 
Scientific Research Publishing (SCIRP) is one of the largest Open Access journal publishers. It is currently publishing more than 200 open access, online, peer-reviewed journals covering a wide range of academic disciplines. SCIRP serves the worldwide academic communities and contributes to the progress and application of science with its publication.

Other selected journals from SCIRP are listed as below. Submit your manuscript to us via either submit@scirp.org or Online Submission Portal.
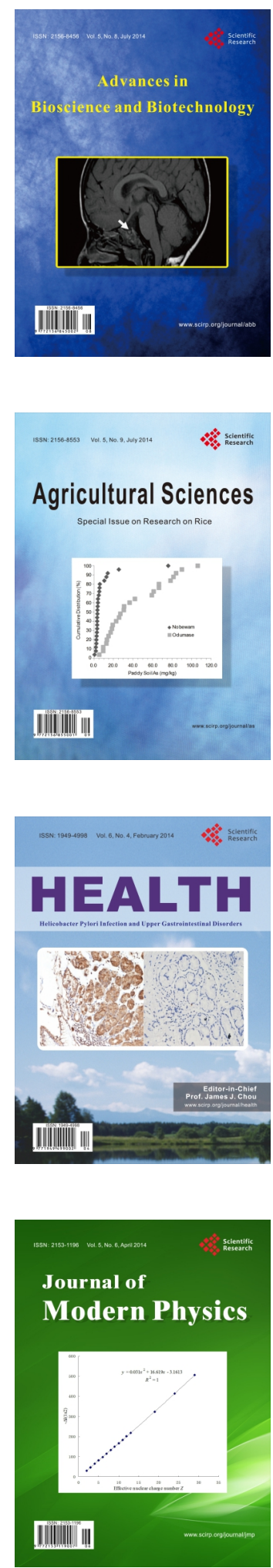
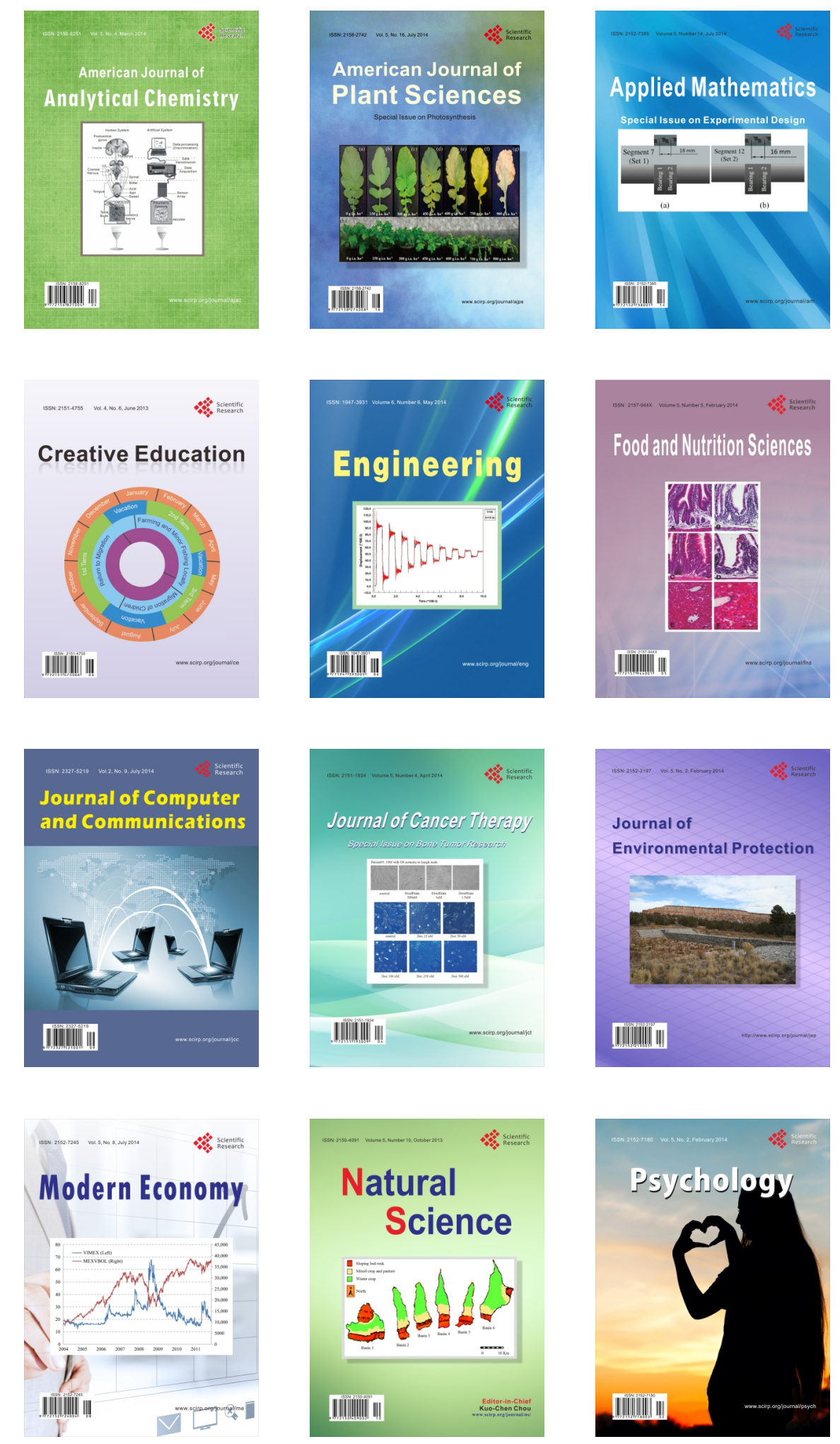